\title{
Flexoelectricity in Nematic and Smectic-A Liquid Crystals
}

\section{Citation}

Prost, Jacques, and Peter S. Pershan. 1976. Flexoelectricity in nematic and smectic-A liquid crystals. Journal of Applied Physics 47(6): 2298-2312.

\section{Published Version}

doi:10.1063/1.323021

\section{Permanent link}

http://nrs.harvard.edu/urn-3:HUL.InstRepos:10361967

\section{Terms of Use}

This article was downloaded from Harvard University's DASH repository, and is made available under the terms and conditions applicable to Other Posted Material, as set forth at http:// nrs.harvard.edu/urn-3:HUL.InstRepos:dash.current.terms-of-use\#LAA

\section{Share Your Story}

The Harvard community has made this article openly available.

Please share how this access benefits you. Submit a story.

Accessibility 


\title{
Flexoelectricity in nematic and smectic-A liquid crystals*
}

\author{
Jacques Prost ${ }^{\dagger}$ and P. S. Pershan \\ Division of Engineering and Applied Physics, Harvard University, Cambridge, Massachusetts 02138
}

(Received 17 November 1975; in final form 27 February 1976)

Flexoelectric effects are observed in both the nematic and smectic-A phases of $p$-butoxybenzal- $p$ - $(\beta$ methylbutyl) aniline (BBMBA) and $p$-cyano-benzylidine- $p$-octyloxyaniline (CBOOA). This is the first reported observation of flexoelectricity in smectic phases. The use of a symmetric interdigital electrode in the homeotropic geometry facilitated the unambiguous separation of linear and quadratic electro-optic effects. Both the interdigital electrodes and those liquid-crystal deformations that are quadratic in the voltage act as optical diffraction gratings with a spacing that corresponds to the repeat distance $d$ for adjacent electrodes. In contrast linear electro-optic effects give rise to diffraction gratings with twice this spacing since adjacent electrodes have opposite voltages. Diffraction maxima due to the linear effects are halfway between the maxima due to the other effects. Using optical heterodyne detection, the intensity of the diffraction maxima believed to arise from the linear effect are indeed observed to be linear in the applied voltage $V(\omega)$. With homodyne detection the diffracted intensity is proportional to $V(\omega)^{2}$. Although previous discussions of flexoelectricity in nematics have been in terms of two flexoelectric coefficients $e_{11}$ and $e_{33}$, we present theoretical arguments that as long as $\nabla \times \mathbf{E}=0$ there is only one true volume coefficient and that the other constant can always be included in surface effects. Our measurements of the volume coefficient $f=e_{11}+e_{33}$ are an order of magnitude larger than previously obtained values for $e_{11}$ and $e_{33}$. Measured values of $f$ are also nearly independent of temperature, in contrast to previous theoretical models, and of similar magnitude in the smectic and nematic phases. Measurements of flexoelectric signals versus the frequency of the driving voltage obtain relaxation times for splaylike nematic fluctuations and undulation-type smectic fluctuations.

PACS numbers: $81.55 .+\mathrm{x}, 78.20 . \mathrm{Jq}$

\section{INTRODUCTION}

Following Meyer's suggestion that there can be a linear coupling between nematic curvature and external electric fields, ${ }^{1}$ there have been only a few attempts to demonstrate the effect. Experiments by Haas et al. ${ }^{2}$ and also by Schmidt et al. ${ }^{3}$ were interpreted in terms of the linear coupling mechanism of Meyer, but these interpretations are not universally accepted. Some indication of the current state of affairs is contained in

De Gennes's discussion of flexoelectricity ${ }^{4}$ (the name he assigned to the linear effect). In his book on liquid crystals he reviews the theory and then remarks that the effect has "apparently been observed". The principal purpose of this paper is to describe an experimental technique that we believe unambiguously proves the existence of flexoelectric coupling in both the nematic and smectic phases of the liquid crystals $p$-butoxybenzal- $p-(\beta$-methylbutyl) aniline (BBMBA) and $p$-cyanobenzylidene- $p$-octyloxyaniline (CBOOA). Since the numerical values we obtain for the flexoelectric coupling parameter is only a factor of 10 larger than the numerical values obtained from previous experiments, the interpretation of these previous experiments in terms of flexoelectricity may very well be correct.

Aside from this principal objective, there are a number of other points that we wish to make. First, concerning the phenomenological theory of flexoelectricity, we will argue in Sec. II that, although previous theories introduce two coefficients, there is only one coefficient appropriate to the bulk flexoelectric effect. The difference arises from the difficulty in separating surface from volume effects. Although it is conceivable that a set of experiments on different liquid crystals with different bounding surfaces might be capable of demonstrating experimentally that it is convenient to treat some surface effects in terms of an equivalent-volume free-energy density, that has not yet been demonstrated and until then we believe the point made in Sec. II should be accepted.

Section III contains a general description of our experimental technique. Most experimental light scattering studies on liquid crystals set out to measure thermodynamic parameters by studying thermally excited fluctuations in equilibrium properties. The alternative to that technique that we describe here is to disturb the system from homogeneous thermodynamic equilibrium by a specific applied force and observe the system response by light scattering. The fluctuation dissipation theorem teaches us that the same information is available from either technique, but it does not say anything about the practical ease of using one or another. In regard to the flexoelectric effect there is no question but that the magnitude of the appropriate response function is easier to measure by the techniques described below.

Section IV deals with specific theoretical problems necessary to the interpretation of the experimental results and Sec. V discusses the quantitative results on the magnitude of the flexoelectric coefficient. Some final remarks on flexoelectricity are contained in Sec. VI.

\section{FLEXOELECTRICITY}

Previous publications have made the point that the elastic-hydrodynamic properties of liquid crystals follow from the elastic-hydrodynamic properties of crystals on taking account of the fact that the increased spatial symmetries of the liquid-crystal phases causes certain of the "first-order" elastic constants that are nonzero in the crystal to vanish in the liquid-crystalline phase. ${ }^{5-7}$ The argument can be extended to include electro-optical and electroelastic effects. To avoid the complications of tensor notation, we present the argument schematically. Following Martin, Pershan, and Swift, ${ }^{5}$ the elastic free-energy density for a crystal in the absence of any external electric fields can be written 


$$
F=\frac{1}{2} B(\nabla u)^{2}+\frac{1}{2} K(\nabla \nabla u)^{2},
$$

where $u$ represents the usual lattice displacement variables of a crystal. The constant $B$ is the conventional fourth-rank elastic tensor and $K$ is a sixth-order tensor that represents "higher-order" or Frank-Oseen elasticity. In crystalline materials the effects of $K$ are completely obscured by the effects of $B$. If one neglects relaxation effects, this representation can be applied to nematics by taking $B \rightarrow 0$, director fluctuations $\delta n \sim \nabla u$, and the $K$ are the Frank constants. ${ }^{5}$ Although the application to nematics when relaxation effects are included is somewhat subtler, it has been discussed by Forster et al. ${ }^{6}$ and also by Martin, Parodi, and Pershan. ${ }^{7}$ For smectics some of the elements of the fourth-rank tensor $B$ vanish, but there are at least three nonvanishing elements for smectic-A phases.

For crystals that lack a center of inversion symmetry, the lowest-order coupling between electric fields and strains are the piezoelectric terms proportional to $E \nabla u$ where $E$ is the electric field. Except for the recently observed chiral smectic phases, ${ }^{8}$ all liquid-crystal phases possess an inversion center and the lowest-order linear coupling to the field $E$ has the form of either $E \nabla \nabla u$ or $\nabla E \nabla u$. These terms are also present in crystals but with nonvanishing first-order elastic constants $B$ the electrically induced strains are proportional to $B^{-1}$ $\nabla E$ and are small for the usual external fields that vary slowly on an atomic scale. In the nematic phase the only nonvanishing element of $B$ corresponds to the compressibility and induced strains $\delta n \sim \nabla u \sim(K \nabla)^{-1} E$ can become large. Note that the factor $\nabla^{-1}$ is symbolic. It indicates a nonlocal or integral relation between $o n$ and $E$. For nematics this effect was discussed first by Meyer. ${ }^{1}$ Common usage is to call it "flexoelectric" to distinguish it from piezoelectric terms. ${ }^{4}$

Reverting now to the conventional notation for nematics the flexoelectric terms are taken as

$$
\delta F=-e_{11}(\nabla \cdot \mathrm{n}) E_{z}-e_{33}\left[\left(\nabla_{z} n_{x}\right) E_{x}+\left(\nabla_{z} n_{y}\right) E_{y}\right],
$$

where the nematic symmetry axis is taken to be the $z$ axis. In this representation there are two phenomenological flexoelectric coupling parameters $e_{11}$ and $e_{33}$. Equation (2.1) is applicable to a linear theory in which one considers only small deviations of the director on from its average direction along $z$. It is important to appreciate the fact that densities of any kind are never uniquely defined. To put this another way, the energy density is defined in such a way that its integral over the sample volume obtains the correct total energy for the system when it is added to the separately defined surface energies. Thus any two expressions for the free-energy density that differ by a total differential can be considered to be equivalent if their respective surface energies are suitably defined. This argument can be applied to Eq. (2.1). For the case that the electric field $\mathbf{E}=-\nabla \phi$, both terms in Eq. (2.1) can be manipulated into the form $\left[\delta n_{x} \partial E_{x} / \partial z+\delta n_{y} \partial E_{y} / \partial z\right]$ plus surface terms. Aside from surface terms, Eq. (2.1) can thus be put in the form

$$
\delta F=+f\left(\delta n_{x} \frac{\partial E_{x}}{\partial z}+\delta n_{y} \frac{\partial E_{y}}{\partial z}\right)
$$

where $f=e_{11}+e_{33}$. The principal conclusion is that there is only one physically independent static flexoelectric coefficient in the nematic phase. As long as $\nabla \times E=0$ the electric field can be derived from a potential and all forms obtainable from Eq. (2.2) are equivalent.

De Gennes discussed flexoelectricity in the smecticA phase in terms of three coefficients $e_{1}=e_{11}, e_{2}=e_{22}$ and $e_{3}=e_{33} \cdot{ }^{9}$ Taking $u$ as the layer displacement variable, if $-\nabla_{x} u$ and $-\nabla_{y} u$ are substituted into $\mathrm{Eq}$. (2.1) for $\delta n_{x}$ and $\delta n_{y}$, respectively, one obtains two of De Gennes's three terms. The additional one has the form $\left(\partial^{2} u / \partial z^{2}\right) E_{z}$. We will argue below that negligible errors result from neglect of the flexoelectric contribution to the displacement vector and taking $\nabla \cdot \epsilon \mathbf{E}=0$. With this approximation, it is straightforward to convert this term into the same form as Eq. (2.2). Thus, even in the smectic phase there is only one bulk flexoelectric coefficient $f \approx e_{11}+e_{33}-e_{22}\left(\epsilon_{1}^{0} / \epsilon_{11}^{0}\right)$ where the superscript $\epsilon$ implies the low-frequency dielectric constant at zero strain.

Although it is possible that for either short wavelengths or very near the nematic-to-smectic phase transition the substitution might be inappropriate, we neglect complications of this type in the following analysis.

The magnitude of $f$ might be crudely estimated from its dimensions of charge/unit length as one electronic charge $\left(4.8 \times 10^{-10}\right.$ esu) divided by a molecular length of $20 \AA$, that is, $f=2.4 \times 10^{-3}$ esu cm$^{-1}$. This is considerably larger than an estimate obtained by dividing the expected dipole moment of typical nematic molecules by the square of the molecular length. This obtains numbers of the order of $f^{\sim} 10^{-5} \mathrm{esu} \mathrm{cm}^{-1}$ that agree with predictions of both Helfrich ${ }^{1}$ and Meyer. ${ }^{1}$ The experimentally determined number that we obtain here is midway between these two values, of the order of $3 \times 10^{-4}$ esu $\mathrm{cm}^{-1}$.

\section{EXPERIMENTAL CONSIDERATIONS}

The measurements to be described here consist of applying a voltage $\phi\left(\omega, q_{z}\right)$ that is periodic in both time (angular frequency $\omega$ ) and one spatial direction (spatial frequency $q_{x}$ ) to an aligned liquid-crystal sample and measuring the light-scattering effects due to this voltage. The combination of Eq. (2.2) and the conventional linear elastic theory for either nematic or smectic liquid crystals leads to the prediction that the optical frequency dielectric tensor will be modulated at the same spatial and temporal frequencies as the voltage. In contrast a second mechanism for coupling the electric potential to optical effects is through dielectric alignment. ${ }^{4}$ This phenomena is quadratic in the applied voltage and leads to modulations in the optical dielectric tensor at $\left(0,2 \omega, 0\right.$ and $\left.2 q_{x}\right)$. The two effects are easily separable in the geometry to be described below.

A periodic electrostatic potential is realized by means of the interdigital electrodes illustrated in Fig. 1. They were very graciously made for us by S. Bernacki and H. Smith of the MIT Lincoln Laboratories. Using optical lithographic techniques, it was possible to put the metallic stripe pattern illustrated in the figure 


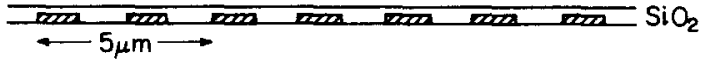

GLASS SLIDE

(a)

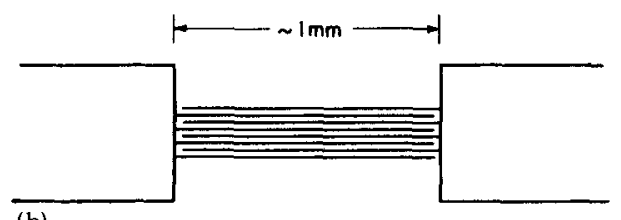

(b)

FIG, 1. Interdigital electrodes: (a) side view, (b) top view.

on a glass slide. An $\mathrm{SiO}_{2}$ layer was then put down on top of the electrodes. Application of a voltage $V(\omega)$ to the opposite electrodes produces a voltage $\phi\left(\omega, q_{x}\right)$ with $2 \pi q_{x}^{-1} \approx 5 \mu \mathrm{m}$ in the center of the pattern. Although the voltage is not really sinusoidal in space, the effects of this can be separated experimentally and will be discussed later. The scattering effects due to $\phi\left(\omega, q_{x}\right)$ are illustrated in Fig. 2 for a monochromatic plane wave (wavelength $\lambda$ ) normally incident on the glass slides. As a result of the linear coupling term [Eq. (2.2)], the director will be tilted in the manner illustrated in Fig.

o)

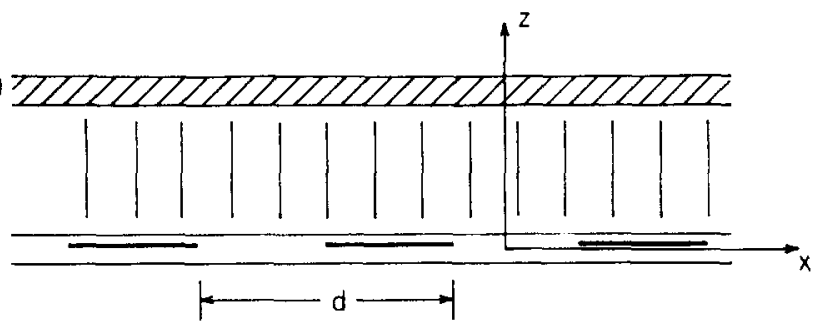

b)

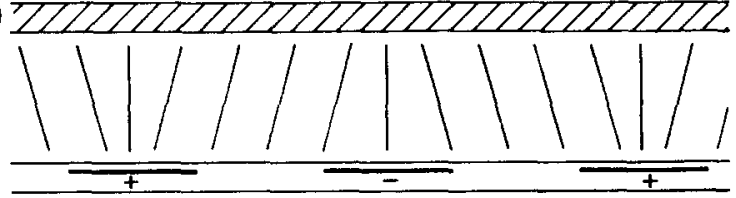

c)

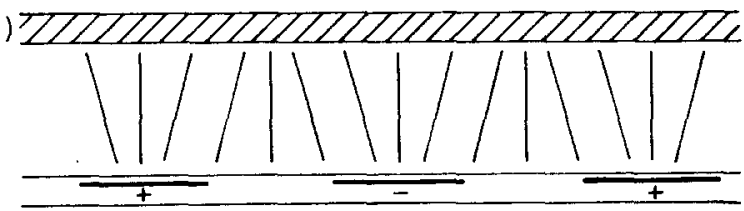

d)

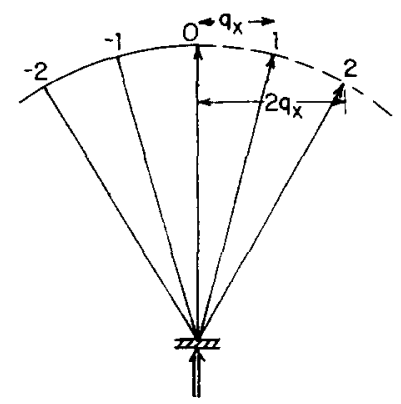

FIG. 2. Schematic illustration of the sample and electrodes: (a) unperturbed sample homeotropic geometry with $V=0$; (b) distortion expected with a linear coupling $(V \neq 0)$; (c) distortion expected with a quadratic coupling $(V \neq 0)$; and (d) direction: 0-incident beam, \pm 1 -light scattered from linear flexoelectric effect, \pm 2 -light scattered from the quadratic dielectric alignment effect. 2(b). Due to the optical anisotropy of the liquid crystal, this results in an optical perturbation with wave vector $q_{x}$ that diffracts the incident wave into directions $\theta$ $=\sin ^{-1}\left( \pm q_{x} \lambda / 2 \pi\right)$. In contrast the quadratic, or dielectric alignment effects, illustrated in Fig. 2(c) produce an optical inhomogeneity that varies as $2 q_{x}$ and which diffracts light at $\theta=\sin ^{-1}\left( \pm q_{x} \lambda / \pi\right)$.

The metallic electrodes themselves diffract light, but if one masks off the "ends" of the electrodes, this light is concentrated at the diffraction maxima for the quadratic effect. Under these circumstances, the only light at $\theta=\sin ^{-1}\left( \pm q_{x} \lambda / 2 \pi\right)$ is due to the linear effect. On the other hand by allowing some light to diffract from the ends of the electrodes, it is possible to obtain a controlled amount of background light at the diffraction maxima of the linear effect and this can be used as a reference wave to obtain heterodyne detection. Higherorder diffraction from the metallic electrodes represent a slight complication to the quantitative interpretation of the results but, as we will discuss below, it is not serious.

In practice the incident light is not usually normal to the glass slides. Figure 3 illustrates the general geometric conditions. Maintaining the axis defined in Fig. 2 relative to the electrodes, the wave vector $\left(k_{i}\right)$ of the incident laser beam is at an angle $\beta^{i}$ with respect to the $z$ axis. If $\mathbf{k}^{i}$ is projected onto the plane of the electrodes, its projection $\mathrm{k}_{p}^{i}$ makes an angle $\alpha^{i}$ with the $x$ axis. By

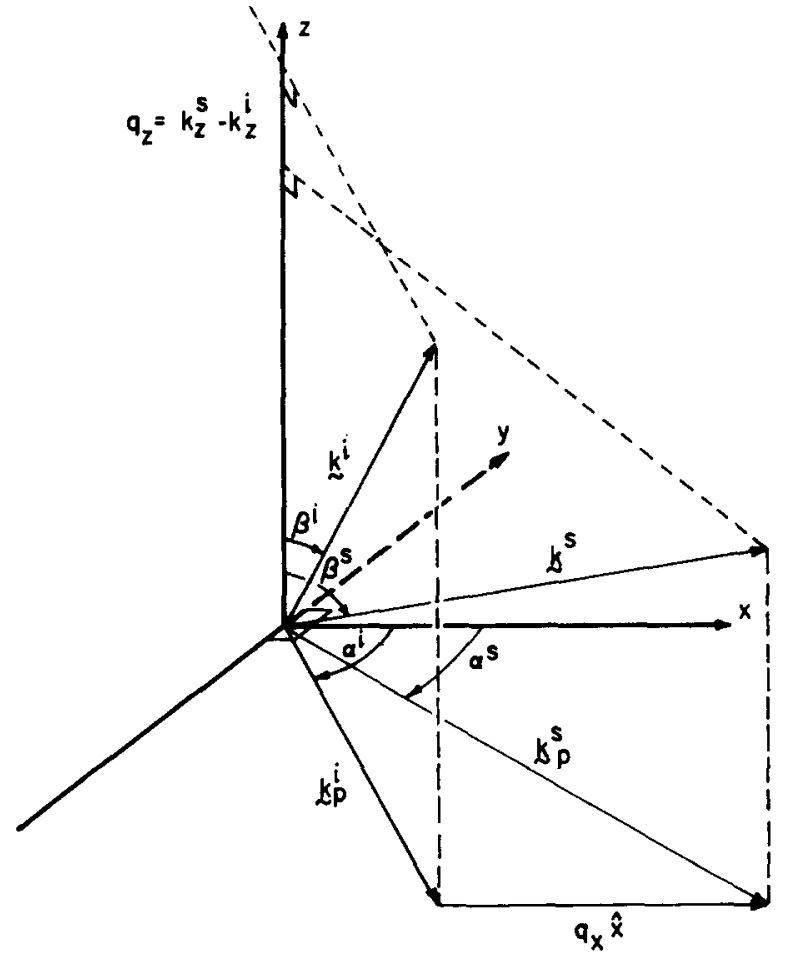

FIG. 3. Scattering geometry: $\mathbf{k}_{i}$ is the wave vector of the incident beam inside the sample, $k_{s}$ is the wave vector of the scattered beam inside the sample. The magnitude of those vectors is equal to $n k_{0}$, where $n$ is the index of refraction for the specific propagation and polarization directions being considered. $\mathrm{q}=q_{x} \hat{x}+q_{z} \hat{z}$ is the wave vector of the Fourier component of the electric field responsible for the scattering. For the linear flexoelectric effect $q_{x}=2 \pi d^{-1}$, where $d$ is defined in Fig. 2 。 


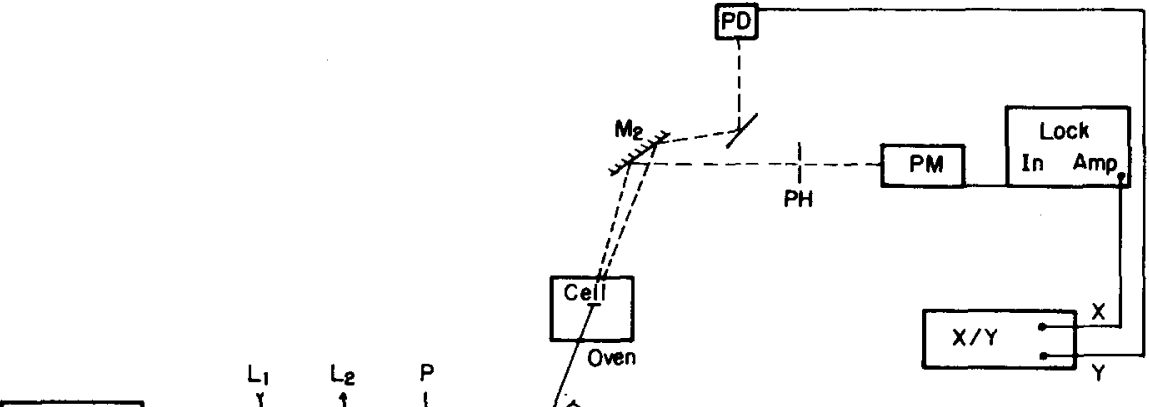

FIG. 4. Block diagram of experimental apparatus. The laser beam is slightly focussed on the cell, through the lenses $L_{1}$ and $L_{2}$, and polarized by $P$. The intensity in the zero order of diffraction is monitored with the photodiode PD. The pinhole PH allows the selection of $\mathbf{k}^{s}$, and the photocurrent given by the phototube PM is analyzed in the lock-in amplifier. The ratio of the two signals is obtained using a digital voltmeter as described in the text. virtue of the periodic electrodes there will be an optical inhomogeneity $\delta \epsilon(q)$ whose spatial Fourier components will only be nonzero for $\mathrm{q}$ in the $x z$ plane. Thus there will be scattered light with wave vector $\mathrm{k}^{s}$ that makes an angle $\beta^{s}$ with the $z$ axis and whose projection on the $x y$ plane $\mathrm{k}_{p}^{s}$ makes an angle $\alpha^{s}$ with the $x$ axis. Since the scattering condition fixes $\mathrm{k}_{p}^{s}=\mathrm{k}_{p}^{i} \pm q_{x} \hat{x}$, and since the scattering is elastic for all practical purposes, the magnitude of $\mathbf{k}^{s}$ is fixed by the polarization of the scattered radiation and the laser frequency. Fixing both the magnitude of $k^{s}$ and its projection $k_{p}^{s}$ allows just one value for $q_{z}=k_{z}^{s}-k_{z}^{i}$. Thus by varying either $\beta^{i}$ or $\alpha^{i}$ it is possible to probe the dependence of $\delta \epsilon$ on $q_{z}$ for fixed $q_{x}$. Note that the only component of $\delta \in$ to vary linearly with $\phi(\omega, q)$ is $\delta \epsilon_{x z}$.

The actual experimental details are illustrated in Fig. 4. The incident wave is obtained from a Spectra Physics $133 \mathrm{He}-\mathrm{Ne}$ laser. The beam is focused onto the electrodes with a small solid angle such that the spread in wave vectors $\mathrm{k}^{i}$ at the electrodes does not exceed the uncertainty in $\mathbf{k}^{i}$ due to the finite beam size. The scattered light is detected by an RCA 1 P28 photomultiplier that is masked by a pinhole in order to define the scattered wave vector $\mathbf{k}^{s}$. For homodyne detection of the flexoelectric signal, the ends of the electrodes are masked off and the only light detected at the appropriate angle is the light scattered by the flexoelectric effect. If the voltage is modulated at a frequency $\omega / 2 \pi$, the homodyne signal $I_{1}(2 \omega)$ is at twice this frequency. For heterodyne detection a small part of the edge of the electrode pattern is exposed to the incident light. This region scatters light into exactly the same direction as the flexoelectric effect and a signal $I_{1}(\omega)$ proportional to $E_{\text {ret }} E_{\text {scat }}(\omega) \cos \phi$ is observed. The angle $\phi$ is the optical phase difference between the reference light $\left(E_{\text {ref }}\right)$ and the flexoelectric signal $E_{\text {scat }}(\omega)$. Although the phase angle is not adjustable, it can be measured by comparing $I_{1}(\omega), I_{1}(2 \omega)$, and the intensity of the ref erence beam.

The photomultiplier output is fed into a PAR phase sensitive detector in which the reference signal is locked to either the voltage applied to the electrodes [to measure $I_{1}(\omega)$ ] or its second harmonic [to measure $I_{1}(2 \omega)$ ]. The intensity of the direct or undeflected beam $I_{1}$ is monitored by UDT 500 photodiode and the ratio $I_{1} / I_{0}$ is obtained from a Data Precision 2540 A1 digital voltmeter in the ratio mode. The sample itself is in the homeotropic geometry and it is sandwiched between the top surface of the electrodes (Fig. 1) and a second glass slide. The sample thickness is $500 \mu \mathrm{m}$. The entire simple assembly is contained in a two-stage oven in which the temperature is controlled to a few $\mathrm{m}^{\circ} \mathrm{C}$.

The first and most basic qualitative observation is the appearance of the expected intensity at the angles corresponding to diffraction by $2 \pi q_{x}^{-1}=5 \mu \mathrm{m}$. This signal was observed in both the nematic and smectic phases of BBMBA and CBOOA. Figures 5 and 6 show typical observations of the expected linear and quadratic voltage dependence of the heterodyne signal at $\omega$ and the homodyne signal at $2 \omega$. Detectable effects were observable for voltages as low as $10^{-3} \mathrm{~V}$ and linearity was confirmed over three decades. The qualitative observation that these signals vanish for incident light with $\alpha^{i}=0$ and polarization in the $y$ direction confirm the origin of the scattering as due to $\delta \epsilon_{x z}$.

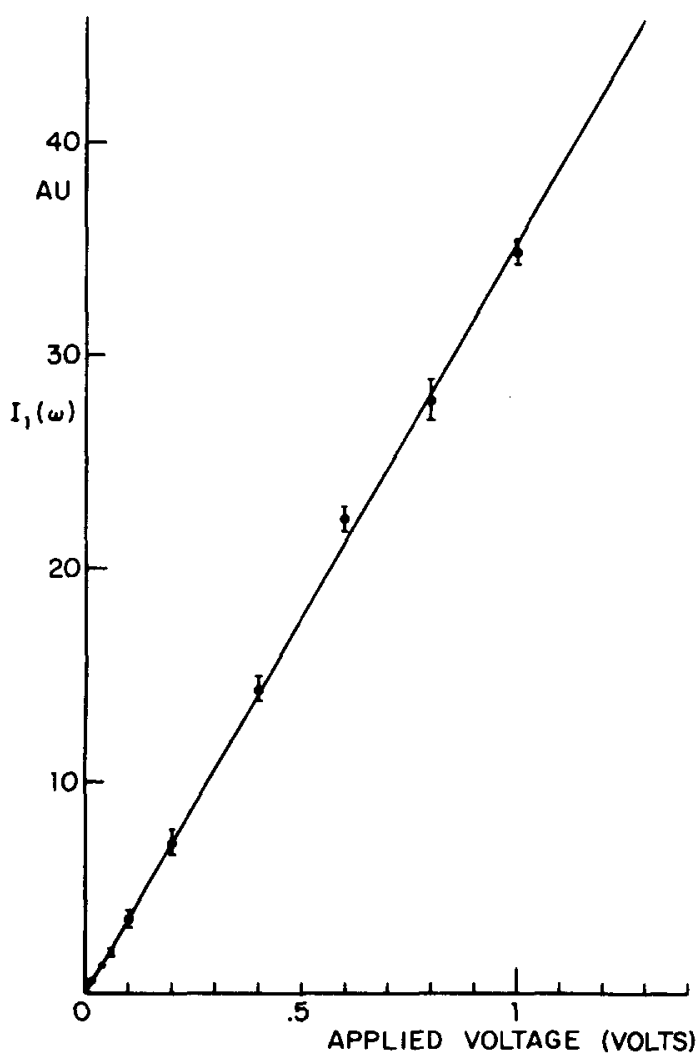

FIG. 5. Typical linear dependence of the heterodyne signal $I_{1}(\omega)$ as a function of the voltage applied on the electrodes. (BBMBA; phase; $T-T_{\mathrm{NA}}=8{ }^{\circ} \mathrm{C} ; T_{\mathrm{NA}}=36^{\circ} \mathrm{C}, \omega / 2 \pi=20 \mathrm{~Hz}$; $E \rightarrow E$ scattering; $\beta^{i}=0$ )。 


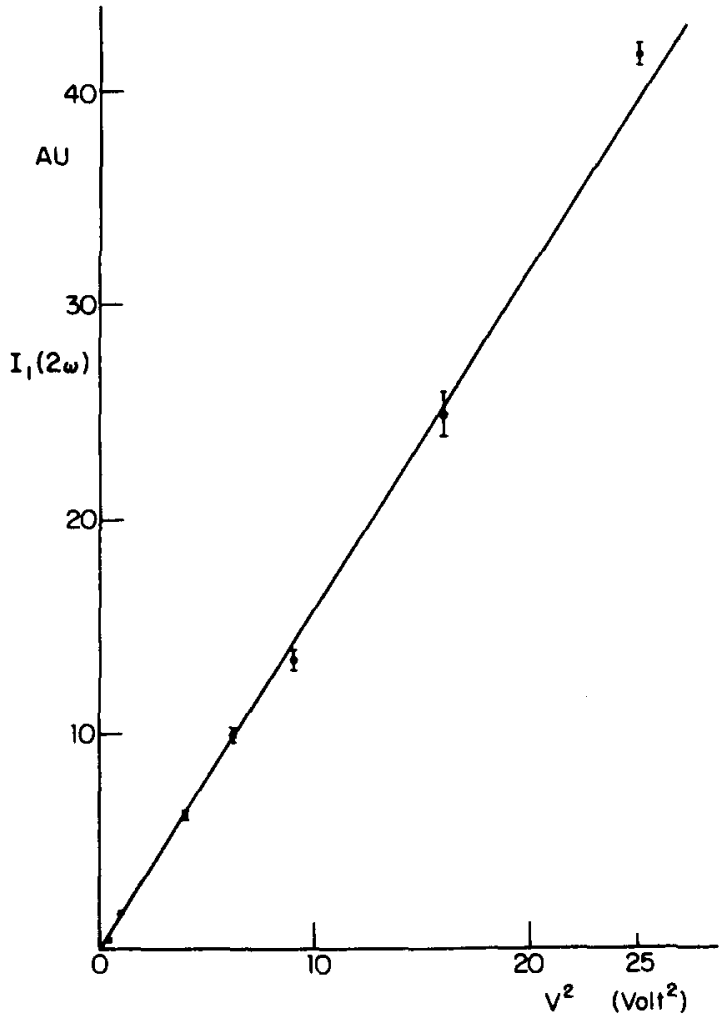

FIG. 6. Typical quadratic voltage dependence of the homodyne signal $I_{1}(2 \omega)$ as a function of the voltage applied on the electrodes (BBMBA; nematic phase; $T-T_{\mathrm{NA}}=8^{\circ} \mathrm{C} ; T_{\mathrm{NA}}=36^{\circ} \mathrm{C} ; \omega /$ $2 \pi=20 \mathrm{~Hz} ; E \rightarrow E$ scattering, $\left.\beta^{i}=0\right)$.

Figure 7 displays typical results for $\left[I_{1}(2 \omega)\right]^{1 / 2}$ versus $\beta^{i}$ when $\alpha^{i}=\pi, \alpha^{s}=0$, and both incident and scattered polarizations are confined to the $x z$ plane. Except for a small discrepancy that we will discuss later, $\left[I_{1}(2 \omega)\right]^{1 / 2}$ approaches zero linearly as $q_{z}$ approaches zero. This is a purely geometric effect due to the $\delta \epsilon_{x z}$ nature of the flexoelectric effect. For incident $E$ fields $E_{x}^{i}, E_{z}^{i}$ the inhomogeneity induces a polarization $4 \pi P_{x}^{s}=\delta \epsilon_{x z} E_{z}^{i}, 4 \pi P_{z}^{s}$ $=\delta \epsilon_{x \varepsilon} E_{x}^{i}$. If we neglect the optical anisotropy for the purposes of making a qualitative argument, we can take $\mathbf{k}^{i} \cdot \mathbf{E}^{i}=0$. For $q_{z}=0$ the scattering condition demands $k_{z}^{i}=k_{z}^{s}$ and thus $k_{x}^{i}=-k_{x}^{s}$. These equations can be combined to show that $\mathbf{k}^{s} \times \mathbf{P}^{s} \approx 0$ or that for this geometry $\mathbf{P}^{s}$ is parallel to $\mathrm{k}^{s}$ and cannot radiate.

The small discrepancy between the condition $q_{z}=0$ and the vanishing of the signal is due to the fact that since $\phi$ is not strictly sinusoidal in $x$, the flexoelectric modulation of incident light that is first diffracted into higher orders by the metallic grating also contribute a small intensity at the observation point. We will show theoretically, however, that near $q_{z}=0$ the only signal linear in $q_{z}=k_{z}^{s}-k_{z}^{i}$ is the one due to flexoelectric modulation of the undiffracted beam. The linear dependence on $q_{z}$ thus provides an experimental method for eliminating effects due to the fact that the voltage is not strictly sinusoidal in $x$. A further confirmation of this would be the results for the smectic phase. Because of the simultaneous presence of both first- and second-order elasticity, the response function for $\delta \epsilon$ is sharply peaked near $q_{z}=0$. In the smectic phase these higherorder diffraction effects will thus be unimportant and in the smectic the intensities should vanish at $q_{z}=0$ to within experimental uncertainty, that is, to within a few mdeg of arc. Although this measurement could not be achieved with BBMBA, Fig. 8 illustrates this effect for CBOOA.

Because of this effect, the geometry with $\alpha^{i}=0$ is not really optimum for studying the flexoelectric effect in the smectic phase. For nonvanishing values of $\alpha^{i}$ incident light polarized as an ordinary wave has a component of $E$ in the $x$ direction proportional to $\sin \alpha^{i}$. The inhomogeneity $\delta \epsilon_{x z}$ produces a polarization in the $z$ direction that radiates as an extraordinary wave in the direction of $\mathbf{k}^{s}$. Thus, in this geometry the flexoelectric effect produces depolarized scattering at a $q_{z}=k_{z}^{s}-k_{z}^{i}$ that depends on $\beta^{i}, \alpha^{i}$, and the average dielectric tensor for the uniaxial material. By rotating the samples around the $z$ axis (i. e., varying $\alpha^{i}$ ), one sweeps out a range of $q_{z}$. The peak intensity, corresponding to $q_{z}$ $=0$, corresponds to scattered light being on the crescent described in Refs. 11 and 12. Figure 9 is a plot of the

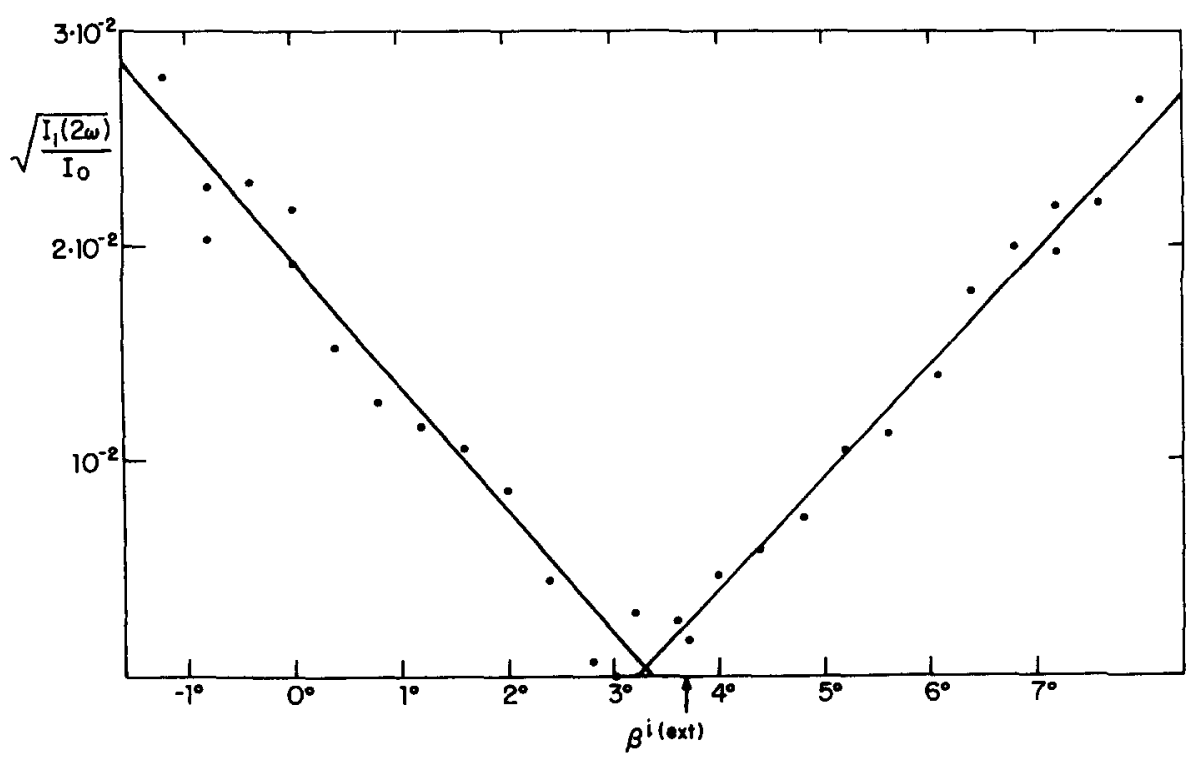

F IG. 7. Dependence of the square root of the homodyne signal on the incidence angle $\left(\beta^{i}\right)^{\text {ext }}$. (BBMBA; nematic phase; $T-T_{\mathrm{NA}}=1{ }^{\circ} \mathrm{C} ; T_{\mathrm{NA}}=34^{\circ} \mathrm{C} ; \omega / 2 \pi=10 \mathrm{~Hz}$; applied voltage: $1 \mathrm{~V} ; E \rightarrow E$ scattering; $\alpha^{i}=\pi ; \alpha^{s}=0$. 


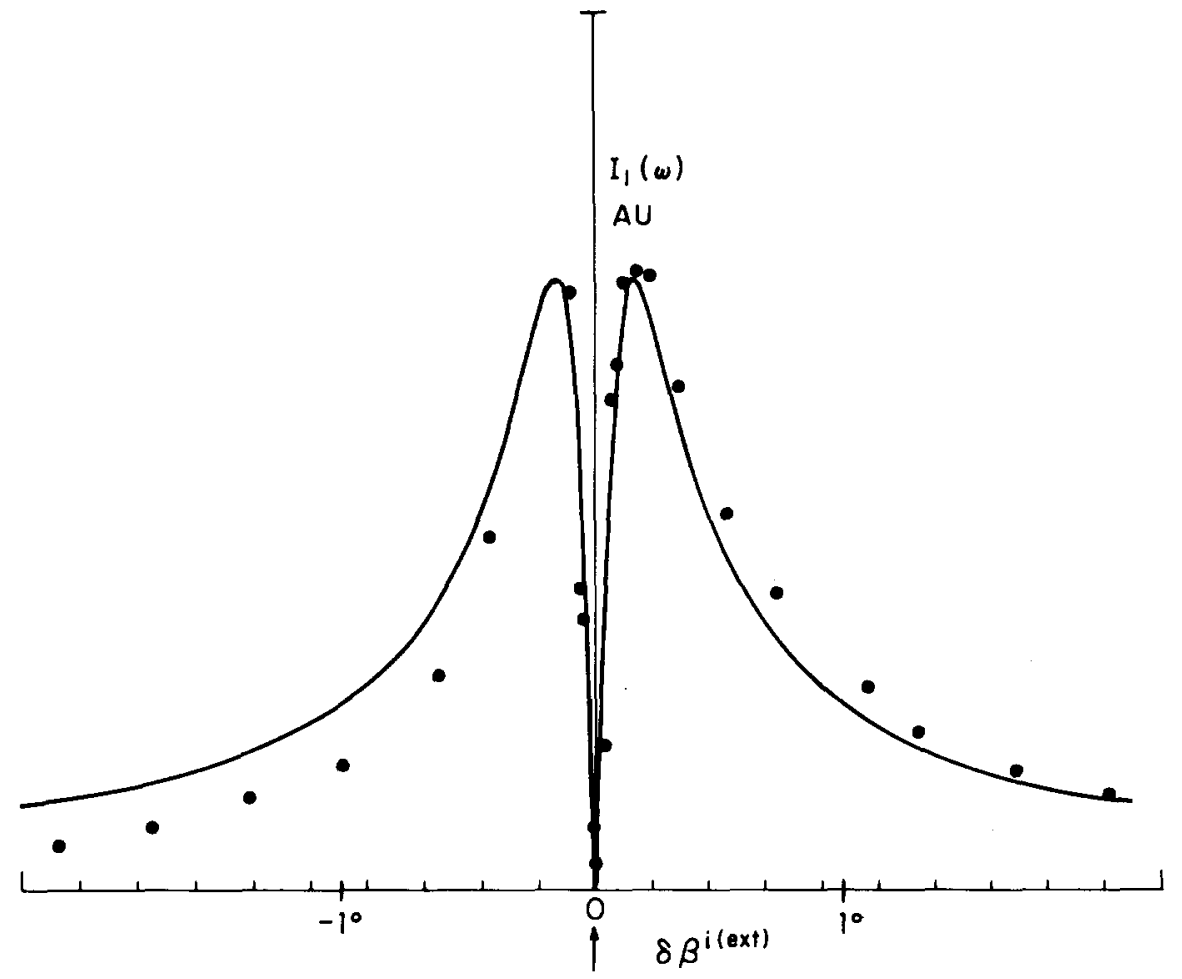

FIG. 8. Dependence of the heterodyne signal $I_{1}(\omega)$ on the incidence angle $\left(\beta^{i}\right)^{\text {ext }}$. (CBOOA; smectic phase; $T_{\mathrm{NA}}$ $-T=0.5^{\circ} \mathrm{C} ; T_{\mathrm{NA}}=81.5^{\circ} \mathrm{C} ; \omega / 2 \pi$ $=100 \mathrm{~Hz}$; applied voltage: $1 \mathrm{~V} ; E \rightarrow E$ scattering, $\alpha^{i}=\pi, \alpha^{s}=0$ ). The arrow shows where the condition $q_{z}=0$ is fulfilled, $\beta^{i}\left(q_{z}=0\right)^{\text {ext }}=3.628^{\circ}$. Note that the signal goes to zero at this very position. The solid curve is simply included as a visual aid. It was chosen to be symmetric. observed signal versus $q_{z}$ in BBMBA. The points are the experimental observations and the solid line is the theoretical response. In the case of Fig. 9 the halfwidth of the curve is determined by the sample thickness. For softer materials this technique would be a sensitive probe of $K / B .{ }^{11,12}$ Note also that the striking features of the angular dependences for the observed signals could be understood in terms of general symmetry or geometric ideas and without any discussion as to whether the driving force was truly a bulk flexoelectric effect or a surface effect in which the bulk distortion was produced by a linear electric effect at the surface. We will elaborate on this point in Sec. IV, but the principal point is that the $q_{z}$ dependence does not really prove the existence of a volume flexoelectric effect.

The frequency dependence of $I_{1}(\omega)$ at fixed temperatures and $q_{z}$ is shown in Fig. 10 for both the nematic and smectic phases of BBMBA. The most striking feature is the order of magnitude of the frequency of which the signal falls off. There is a change by a factor of $10^{3}$ in going from the nematic to smectic phases. Deferring a detailed discussion to Sec. IV, we point out that in the nematic phase the relaxation frequency for a pure splay mode would be $K_{11} q_{x}^{2}\left(\gamma_{1}^{*}\right)^{-1}$, while in the smectic

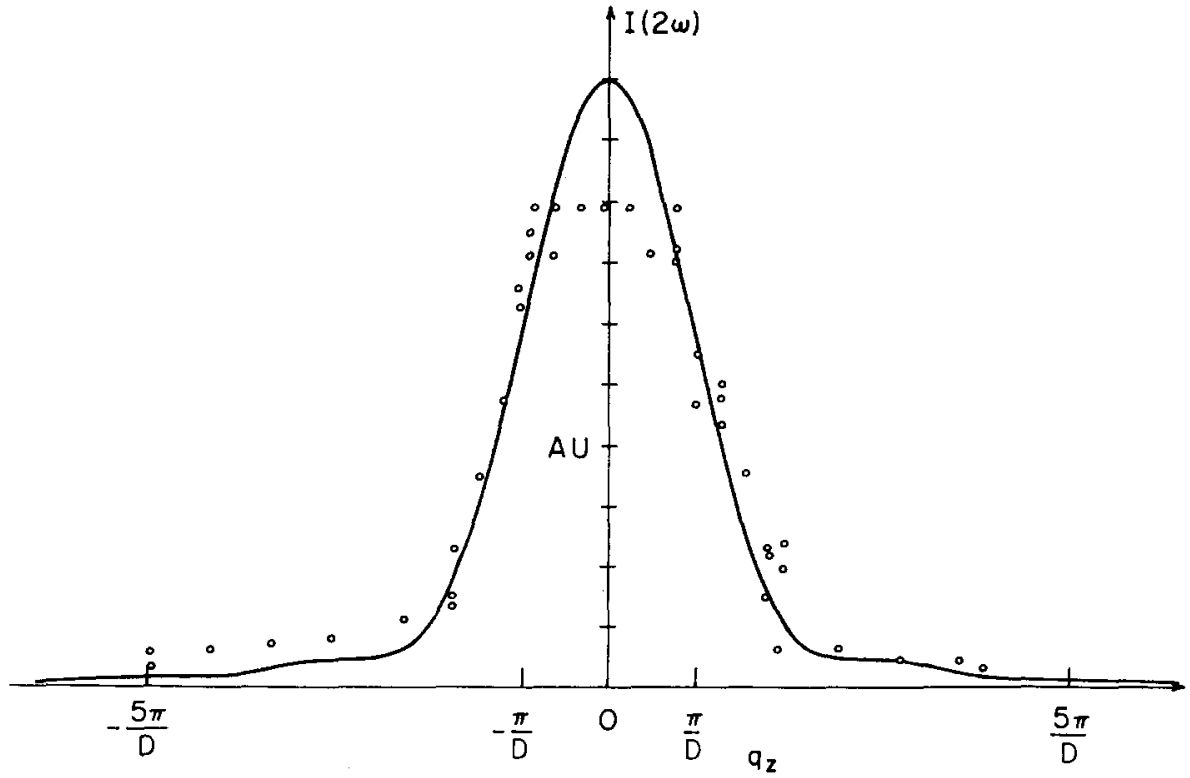

F IG. 9. Homodyne signal in the smectic phase of BBMBA as a function of $q_{z}$; dots are the experimental points; solid line is the theoretical curve for the $q_{z}$ dependence of $\left|\delta n\left(q_{z}\right)\right|^{2}$. See, for example, Eqs. (4.19) and (4.22). (BBMBA; smectic phase; $T_{\mathrm{NA}}-T$ $=0.3{ }^{\circ} \mathrm{C} ; T_{\mathrm{NA}}=34^{\circ} \mathrm{C} ; \omega / 2 \pi=210 \mathrm{~Hz} ;$ applied vol tage $2 \mathrm{~V} ; 0 \rightarrow E$ scattering; $\left(\beta^{i}\right)=0.24 \mathrm{rad}$, the variation of $q_{z}$ is obtained by varying $\alpha^{i}$ around the value $\pi / 2)$. 

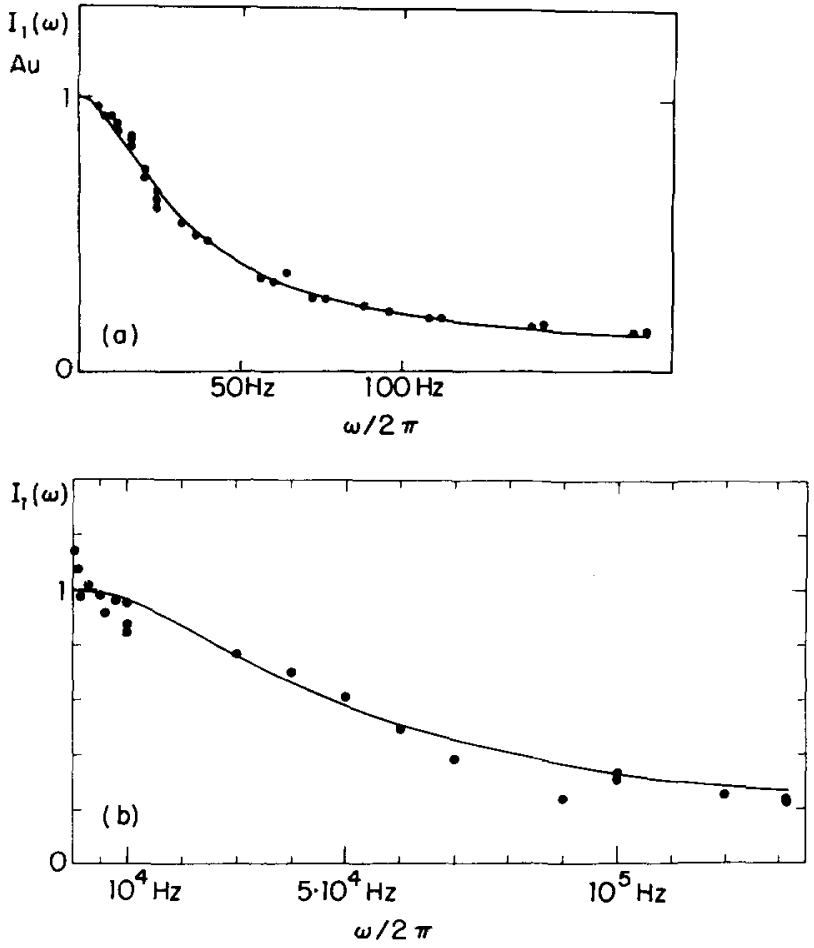

F IG. 10. F requency dependence of the heterodyne signal: (a) $\mathrm{BBMBA}$; nematic phase; $T-T_{\mathrm{NA}} \simeq 7^{\circ} \mathrm{C} ; T_{\mathrm{NA}} \simeq 34^{\circ} \mathrm{C}$; applied voltage: $5 \mathrm{~V} ; 0 \rightarrow E$ scattering; $\left(\beta^{i}\right)^{\mathrm{eat}}=0.24 \mathrm{rad} ; \alpha^{i} \simeq \pi / 2 ;$ dots are the experimental points, solid line; $y=\left[1+(\omega \tau)^{2}\right]^{-1 / 2}$ where $\tau^{-1}=(2 \pi)(27) \mathrm{sec}^{-1}$. (b) BBMBA; smectic phase; $T_{\mathrm{NA}}-T=8^{\circ} \mathrm{C}$; $T_{\mathrm{NA}}=36^{\circ} \mathrm{C}$; the geometrical conditions [identical to those given in (a) $]$ correspond to $q_{z}=0$; dots are the experimental points; solid line is the theoretical curve as given by Eq. (4.19).

the anisotropic elastic effects lead to a value given by $B \pi^{2} \nu^{-1} D^{-2} q_{x}^{-2}$, where $K_{11}$ is the usual Frank constant and $\gamma_{1}^{*}$ is an effective splay viscosity parameter. It is defined explicitly following Eq. (4.11). The quantity $B$ is the smectic elastic constant for layer compression, $\nu$ is

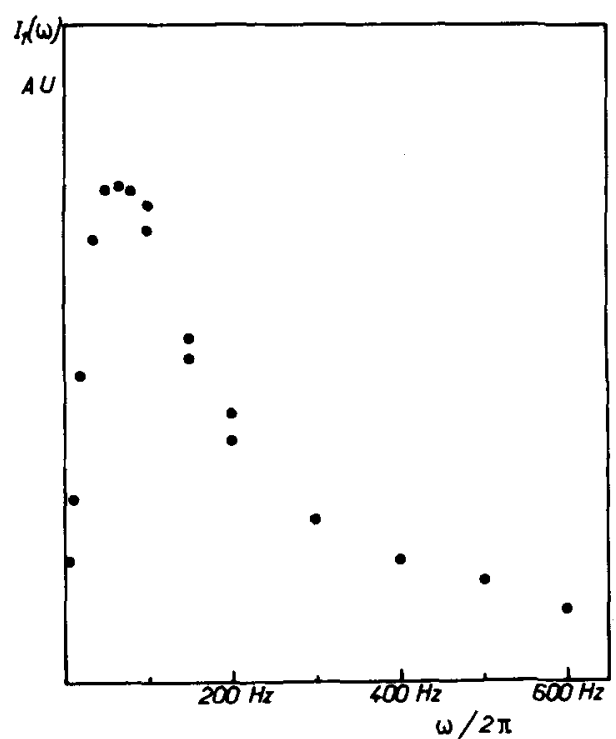

FIG. 11. Low-frequency dependence of the heterodyne signal in the nematic phase of $\mathrm{CBOOA}: T-T_{\mathrm{NA}}=1{ }^{\circ} \mathrm{C} ; T_{\mathrm{NA}}=81.5^{\circ} \mathrm{C}$; $V=1 \mathrm{~V} ; E \rightarrow E$ scattering; $\beta_{i}^{\text {ext }}=0$. a shear viscosity, and $D$ is the sample thickness. Taking $K_{11} \sim 10^{-6}$ dyn at $45^{\circ} \mathrm{C}$ obtains a reasonable value of $\gamma_{1} \sim 0.7 \mathrm{P} .{ }^{13}$ On the other hand, if $\nu$ is taken to be $1 \mathrm{P}$, the deduced value of $B \sim 8 \times 10^{9} \mathrm{erg} \mathrm{cm}^{-3}$ is also reasonable. Equations enabling a quantitative study of the response function versus $\omega$ will be obtained in Sec. IV. These will enable us to draw specific conclusions regarding the boundary conditions in the sample and to obtain a numerical value for $f=e_{11}+e_{33}$. Figure 11 illustrates the low-frequency dependence of the heterodyne signal observed in the nematic phase of CBOOA. Below about $60 \mathrm{~Hz}$ the amplitude falls off in just the manner one would expect from a volume effect if the electric fields within the bulk were being screened by ionic impurities. The relaxation time for this effect can be estimated from the known magnitude of diffusion constants $D$ for small dye molecules in MBBA. ${ }^{14}$ The resultant $D q_{x}^{2} / 2 \pi$ is just the correct magnitude to explain the effect. Similar results were also obtained for BBMBA except that since lower temperatures could be used, the relaxation time was approximately a factor of 10 larger, i. e., the fall off began about 3 or $4 \mathrm{~Hz}$. If we assume that the bulk effect is being screened and the surface effects are not, we can conclude from the shape of the frequency dependence that $f \geqslant 10 p$, where $p$ is the coefficient for surface polarization to be defined below [see Eq. (4.8)].

Figure 12 is a plot of the homodyne $\left[I_{1}(2 \omega)\right]$ signal versus temperature for BBMBA. The heterodyne signal could also be measured under identical conditions and the optical phase angle $\phi$ was found to be essentially constant over this temperature range. Anticipating the theoretical results of Sec. IV $I_{1}(2 \omega)$ is proportional to $\left[\Delta_{\epsilon}\left(f / K_{11}\right)\right]^{2}$ if the observed signal is bulk flexoelectricity. Figure 13 contains a plot of $\left[I_{1}(2 \omega)\right]^{1 / 2} K_{11}(\Delta \epsilon)^{-1}$ versus $T-T_{\mathrm{NA}}$ where the values of $K_{11}$ and $\Delta \epsilon$ were taken from Refs. 15 and 16. In view of the fact that a theoretical model has been proposed in which $f=e_{11}+e_{33}$ was es-

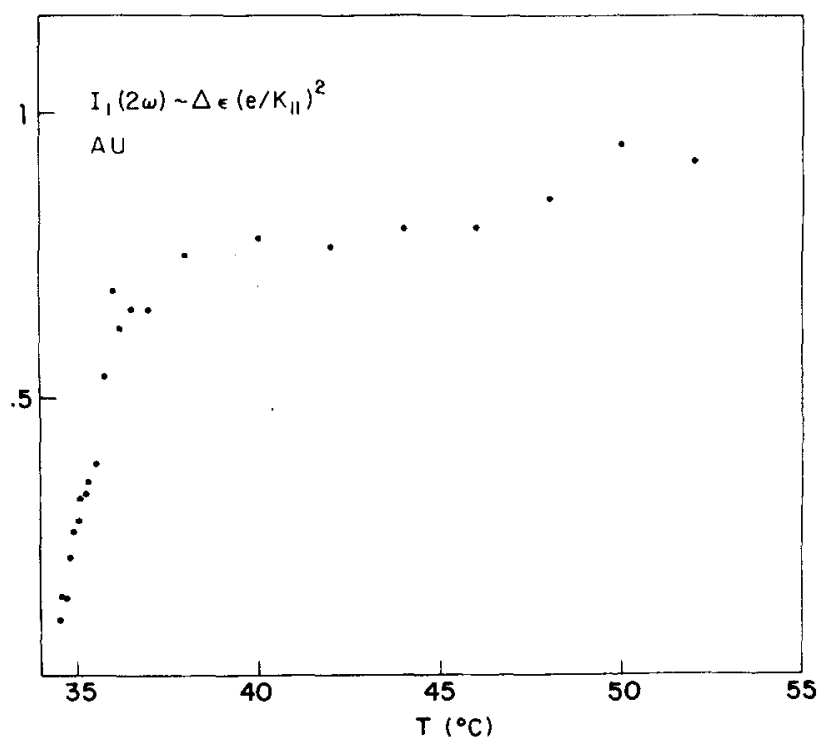

FIG. 12. Temperature dependence of the homodyne signal (BBMBA; nematic phase; $T_{\mathrm{NA}}=34.55^{\circ} \mathrm{C} ; \omega / 2 \pi=10 \mathrm{~Hz}$; applied voltage: $V=0.5 \mathrm{~V}$; same geometrical conditions as in Fig. (10). 


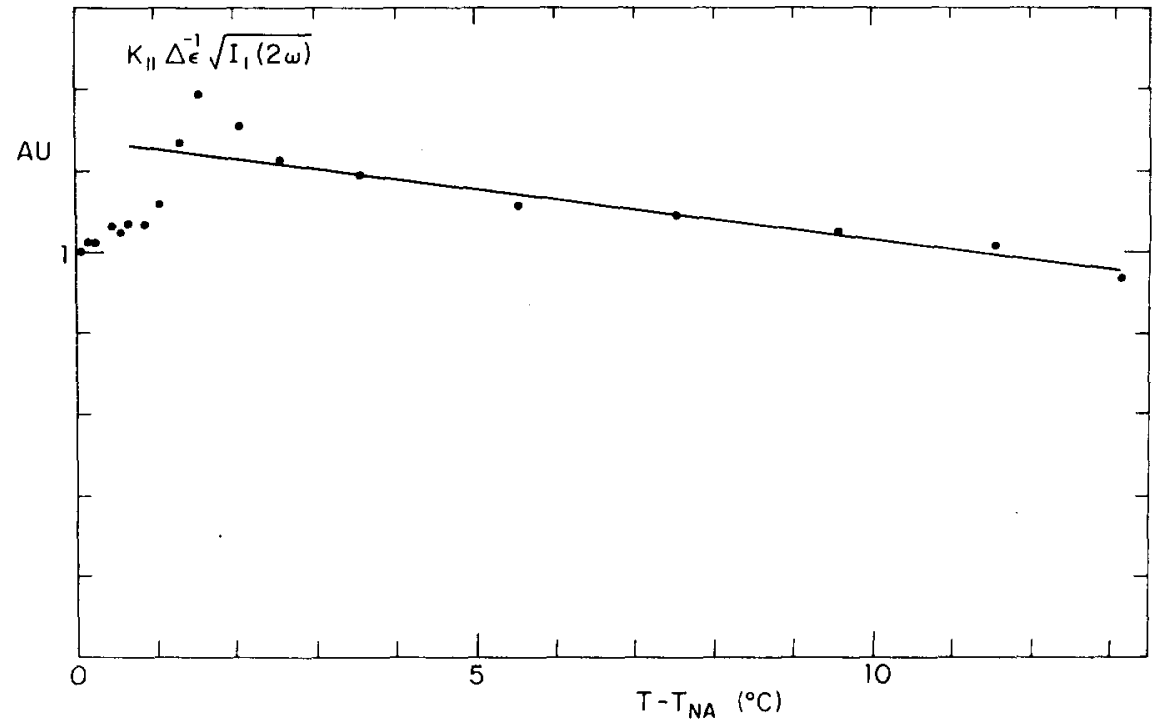

F IG. 13. Temperature dependence of $f \propto K_{11} \Delta \epsilon^{-1}\left[I_{1}(2 \omega)\right]^{1 / 2}$. Same conditions as in Fig. 11.

sentially proportional to the elastic constants, ${ }^{3}$ this result must be examined quite carefully. Both $K_{11}$ and $K_{33}$ have striking temperature dependences but according to this result $f=e_{11}+e_{33}$ varies only slightly.

The temperature dependence of $I_{1}(\omega)$ is shown in Fig. 14 for the smectic phase of BBMBA. One possible uncertainty involves the correct assignment of the nematicsmectic phase transition temperature $T_{\mathrm{NA}}$. The $T_{\mathrm{NA}}$ shown here was identified as that temperature at which one first observed the crescent pattern that is characteristic of static light scattering from smectic materials. Since this pattern is only expected in well-aligned samples for which deviations from perfect alignment are not too large, it is not obvious that this is the correct choice. Nevertheless, it does coincide with the temperature at which the characteristic nematic fluctuations disappear. The problem with this choice of $T_{\mathrm{NA}}$ is that the temperature dependence of the flexoelectric signal does not seem to change at $T_{\mathrm{NA}}$. Rather, the significant decrease in the flexoelectric signal that is predicted to occur when $B q_{z}^{2} q_{x}^{-2} \gg K_{11} q_{x}^{2}$ (see Sec. IV) does not happen until $T-T_{\mathrm{NA}} \approx-0.15^{\circ} \mathrm{C}$. Since the nematicsmectic phase transition for BBMBA is first order, we do not believe this can be attributed to the temperature dependence of $B$. Although a systematic error in temperature could reconcile the two observations, we do not believe our temperature measurements are this bad. A more likely explanation would involve the temperature dependence of both boundary conditions and the ease of nucleating defects in the smectic structure. This difficulty aside, the principal observation is that for $T-T_{\mathrm{NA}}$ $\lesssim-0.15^{\circ} \mathrm{C}$ the flexoelectric signal decreases very rapidly with decrease in temperature. Anticipating the theoretical results in Sec. IV and further experimental results to be presented in Sec. V, this decrease is interpreted as an increase in the smectic elastic constant $B$ with decreasing temperature.

Finally, we would like to describe an interesting effect that should be investigated in more detail. Figure 15 decribes the striking difference between the flexoelectric signal observed in the smectic phase of a new sample and the same sample approximately one month later. During that month, the sample was exposed to normal atmospheric conditions and from past experiience with this compound we might guess that approximately $1 \%$ of the Schiff base molecules have been oxidized. For example, the depression of the transition temperature by approximately $8^{\circ} \mathrm{C}$ corresponds to the drop in the MBBA transition temperature on dissolving approximately $1 \%$ solute molecules in MBBA. The extra signal intensity at low frequencies in the older or aged sample is exactly what would be predicted if the "elastic dipole" density discussed in a previous publication could adjust its elf to lower the effective elastic

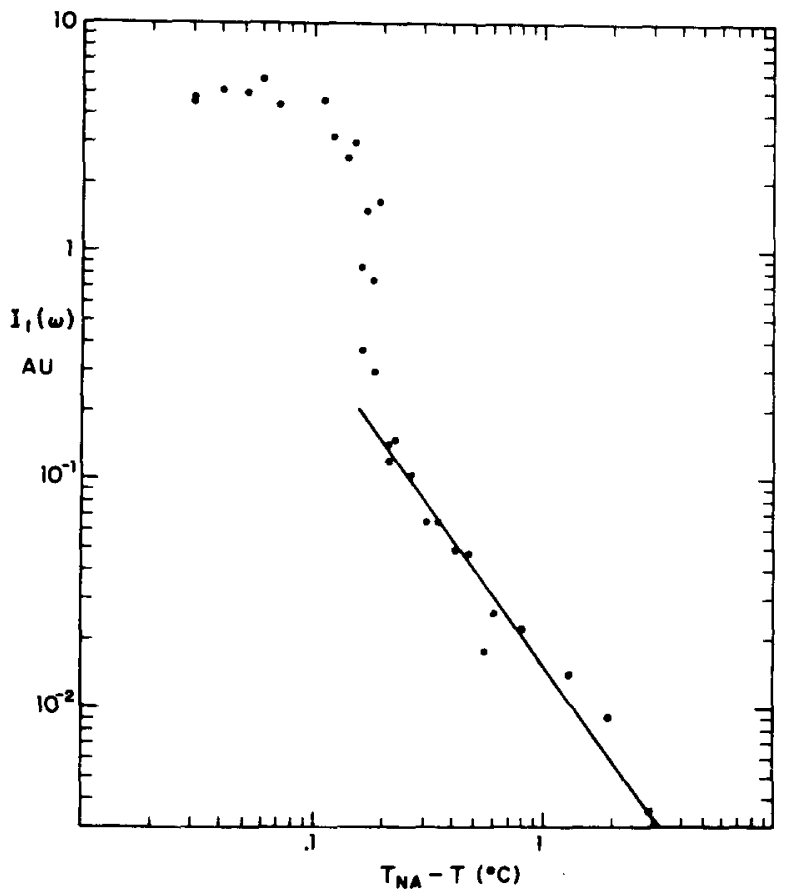

F IG. 14. Temperature dependence of the heterodyne signal (BBMBA; smectic phase; $T_{\mathrm{NA}}=34.65^{\circ} \mathrm{C} ; \omega / 2 \pi=10 \mathrm{~Hz}$; applied voltage $2 \mathrm{~V}$; geometrical conditions of $\mathrm{Fig} .10$ ). 


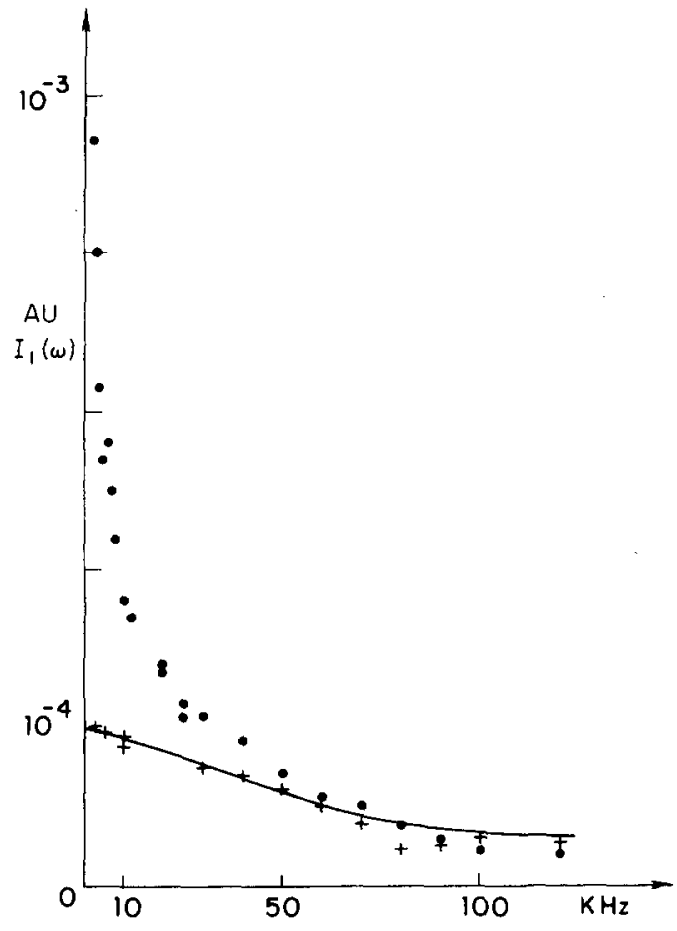

FIG. 15. Comparison of the frequency dependence of $I_{1}(\omega)$ obtained with a fresh sample (crosses) and an aged sample (circles) (BBMBA, room temperature, smectic phase): (a) curve displayed over the whole spectral range and (b) low-frequency part, the solid line corresponds to the expression

$0.95 \omega^{-3}\left[1+(\omega \tau)^{2}\right]^{-1 / 2} \times 10^{-4}$, where the constant term is the one corresponding to the pure sample and $\tau^{-1}=(2 \pi)(900) \mathrm{sec}^{-1}$.

constant and increase the light scattering intensity. ${ }^{17}$ For this interpretation the relaxation time for the "elastic dipole" density would have to be of the order of $10^{-4} \mathrm{sec}$, so that for frequencies above $10^{4} \mathrm{~Hz}$ the elastic dipoles could not move and both aged and new samples would behave identically. This seems somewhat fast if the relaxation mechanism is the diffusion of impurities of the same size as the broken Schiff' $s$ base. On the other hand, elastic dipole relaxation could also arise from either reorientation or other chemical effects. Alternative interpretations involving "elastic monopole" effects could also contribute to the increased low-frequency signal. ${ }^{17}$ There is no point in further speculation since additional experimentation is clearly required.

\section{THEORETICAL DISCUSSION}

There are three separate calculations that must be carried out in order to make quantitative use of the type of data that was illustrated in Sec. III. First, it is necessary to obtain a reasonable description of the electrostatic potential $\phi(x, z)$ that results from the electrodes shown in Fig. 1. Second, we need to calculate the optical inhomogeneity $\delta \epsilon$ that results from this potential if we assume the flexoelectric coupling described by Eq. (2.2). Finally, we must calculate the scattered light intensity due to the predicted $\delta \epsilon$.

\section{A. Electrostatic potential due to interdigital}

Since the electrodes sketched in Fig. 1 are of much greater extent in the $y$ direction than their periodicity in the $x$ direction, we approximate this problem by one of infinite extent along $y$. Also both the thickness of the metallic deposit and the covering $\mathrm{SiO}_{2}$ layer are small compared to the periodicity and we take both of these to be zero. The over-all sample thickness is also larger than the periodicity and we assume the liquid crystal is of infinite extent along the $+z$ direction. For the moment we will also neglect the dielectric anisotropy of the liquid crystal.

We now wish to solve for the potential $\phi$ satisfying $\nabla^{2} \phi=0$ and the following boundary conditions on the regions illustrated in Fig. 16(a):

$$
\begin{array}{ll}
\text { on } \mathrm{AB} \text { and } \mathrm{A}^{\prime} \mathrm{B}^{\prime}: & E_{x}=0 \\
\text { on } \mathrm{A}^{\prime} \mathrm{C}^{\prime}: & \phi=-\frac{1}{2} V, \\
\mathrm{AC}: & \phi=+\frac{1}{2} V, \\
\mathrm{C}^{\prime} \mathrm{C}: & E_{z}=0 .
\end{array}
$$

The last boundary condition is also an approximation
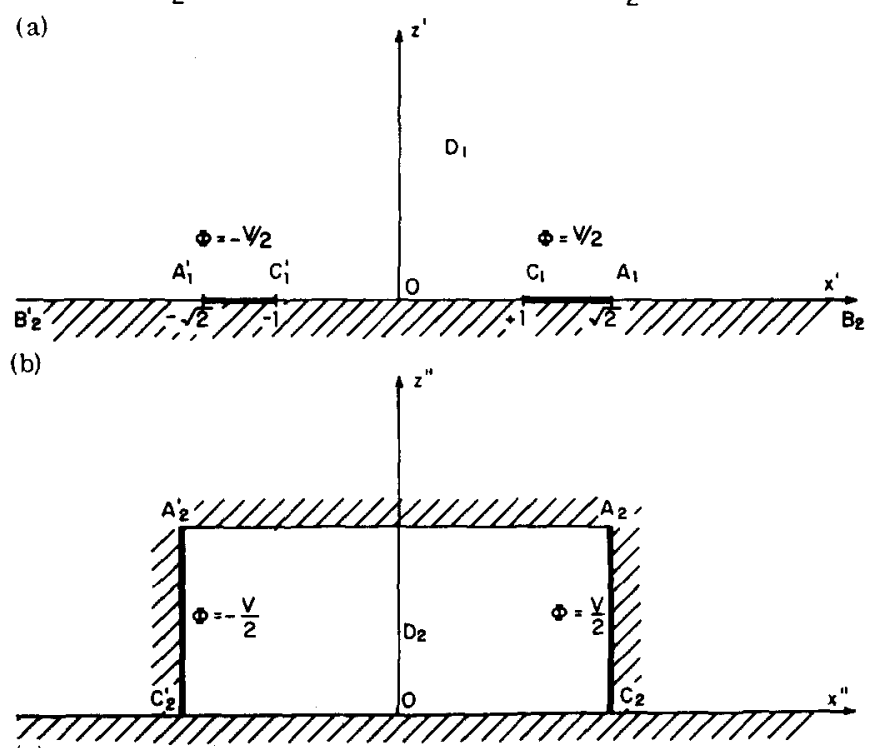

(c)

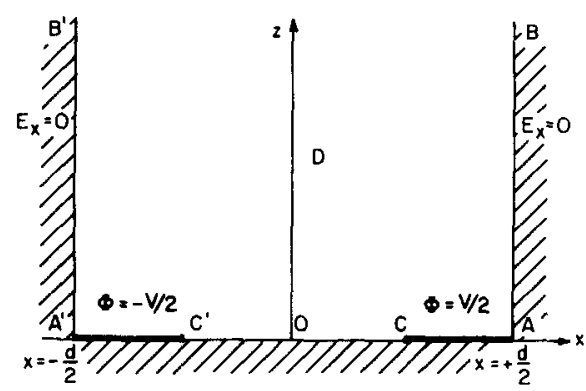

FIG. 16. Successive transformations facilitating solution of the electric field problem. The region in (a) $\mathrm{D}$ is mapped into $\mathrm{D}_{1}$; in (b) by $\xi^{\prime}=2 \sin (\pi \xi / d)$; and $D_{1}$ in $(b)$ is mapped into $D_{2}$ in (c) by $\xi^{\prime \prime}=F\left(1 / \sqrt{2}, \xi^{\prime}\right)$, where the function $F$ is defined in the text. The corresponding boundary conditions are evident on the figure and $\xi=x+i z, \xi^{\prime}=x^{\prime}+i z^{\prime}$, and $\xi^{\prime \prime}+i z^{\prime \prime}$. 
since the regions above and below the electrodes are not equivalent dielectrics. The solution to this problem is a straightforward exercise in conformal mapping. ${ }^{18}$ Using the transformations $\xi=x+i z$ and $\xi^{\prime}=\sqrt{2} \sin (\pi \xi / d)$ followed by $\xi^{\prime \prime}=F(1 / \sqrt{2}, \xi)$, the region in Fig. $16(\mathrm{a})$ is mapped into the interior of the rectangular illustrated in Fig. 16(c). The function $F(k, u)=\int_{0}^{u}\left[\left(1-v^{2}\right)(1\right.$ $\left.\left.-k^{2} v^{2}\right)\right]^{-1 / 2} d v$ is the elliptic integral of the first kind. The electrostatic potential $\Phi$ is the real part of a complex function $\Omega=\Phi+i \psi$ which can be obtained from Fig. 16 (c) as $\Omega=V \xi^{\prime \prime} / 2 G$ where $G=F\left(2^{-1 / 2}, 1\right)$. Thus in terms of the original variable

$$
\Omega=2^{-1} G^{-1} V F\left(2^{-1 / 2}, 2^{1 / 2} \sin (\pi \xi / d)\right)
$$

or in a more explicit form

$$
\Omega=2^{-1 / 2} \pi G^{-1} V d^{-1} \int_{0}^{\xi}[\cos (2 \pi s / d)]^{-1 / 2} d s .
$$

The electric fields are easily obtained in terms of $d \Omega /$ $d \xi$ using the Cauchy relations

$$
E_{x}-i E_{z}=-\left(2^{-1 / 2}\right) \pi G^{-1} V d^{-1}[\cos (2 \pi \xi / d)]^{-1 / 2} .
$$

The electric fields is expected to be periodic in $x=\operatorname{Re} \xi$ with a period of $2 d$. This follows from Eq. (4.3) if one assigns the appropriate signs to the real part of $[\cos (2 \pi \xi / d)]^{-1 / 2}$. In the region $z>0$ a power series expansion of Eq. (4.3) obtains

$$
\begin{aligned}
E_{x}= & -\pi G^{-1} V d^{-1} \sum_{n=0}^{\infty} \frac{\Gamma\left(+\frac{1}{2}\right)}{\Gamma\left(-n+\frac{1}{2}\right) n !} \\
& \left.\times \exp [-4 n+1) \pi z d^{-1}\right] \cos \left[(4 n+1) \pi x d^{-1}\right], \\
E_{z}= & \pi G^{-1} V d^{-1} \sum_{n=0}^{\infty} \frac{\Gamma\left(+\frac{1}{2}\right)}{\Gamma\left(-n+\frac{1}{2}\right) n !} \\
& \times \exp \left[-(4 n+1) \pi z d^{-1}\right] \sin \left[(4 n+1) \pi x d^{-1}\right] .
\end{aligned}
$$

Since $E_{x} \rightarrow 0$ as $z \rightarrow \infty$, the potential $\phi$ is directly obtained from $\phi=-\int_{\infty}^{z} d z E_{z}$. Another interesting quantity in relation to the quadratic dielectric coupling is

$$
\begin{aligned}
E_{x} E_{z}= & -4^{-1} V^{2} \pi^{2} G^{-2} d^{-2} \operatorname{Im}[\cos (2 \pi \xi / d)]^{-1} \\
= & -2^{-1} V^{2} \pi^{2} G^{-2} d^{-1} \sum_{n=0}^{\infty}(-1)^{n} \exp \left[-2 \pi(2 n+1)|z| d^{-1}\right] \\
& \times \sin \left[2 \pi(2 n+1) x d^{-1}\right] .
\end{aligned}
$$

Although the above was derived after explicitly neglecting the dielectric anisotropy of the liquid crystal, that is easily corrected. In place of $\nabla^{2} \phi=0$ one wants the solution $\epsilon_{\perp}^{0} \partial^{2} \phi / \partial x^{2}+\epsilon_{\|}^{0} \partial^{2} \phi / \partial z^{2}=0$ where the superscript distinguishes the low-frequency dielectric tensor $\epsilon^{0}$ from the optical-frequency tensor $\epsilon$. The correct solution is then obtained by substituting $z\left(\epsilon_{\perp}^{0} / \epsilon_{\|}^{0}\right)^{1 / 2}$ for $z$ in the above equations. To be completely rigorous, the effect of including the linear flexoelectric effect on the displacement vector $D$ must also be included. That is, $\nabla \cdot D$ is not simply equal to $-\nabla \cdot(\epsilon \circ \nabla \phi)$ with a constant $\epsilon$. In principle such effects could be included in the definition of $\epsilon$, however the dielectric tensor is often tacitly taken to represent the relation between $D$ and $E$ when $\delta n=0$. Detailed discussions on this point are not warranted here since the corrections of the order of $f^{2} K_{11}^{-1} \sim 10^{-3}$ (where $K_{11}$ is a curvature elastic constant) are negligible. ${ }^{4}$

\section{B. Electric-induced liquid-crystal distortions}

\section{Nematic phase}

Taking the fundamental wavelength $\lambda_{e}=2 \pi\left(q_{x}\right)^{-1}=2 d$ the free energy per unit length in the $y$ direction can be written as the sum of a volume term

$$
\begin{aligned}
F_{v}= & \frac{1}{2} \int_{x_{0}}^{x_{0}+\lambda} d x \\
& \times \int_{0}^{D} d z\left[K_{11}\left(\frac{\partial n}{\partial x}\right)^{2}+K_{33}\left(\frac{\partial n}{\partial z}\right)^{2}+2 f \delta n\left(\frac{\partial E_{x}}{\partial z}\right)\right. \\
& \left.-(2 \pi)^{-1} \Delta \epsilon^{0}(\delta n) E_{x} E_{z}\right]
\end{aligned}
$$

and surface terms $F_{s}$ and $F_{s}^{\prime}$. The constants in $F_{v}$ are the Frank constants $K_{11}$ and $K_{33}$, the flexoelectric constant $f=e_{11}+e_{33}$, and the low-frequency dielectric anisotropy $\Delta \epsilon^{0}=\left(\epsilon_{11}^{0}-\epsilon_{\perp}^{0}\right)$. Since we assume the director distortions are all confined to the $x z$ plane $\delta n \equiv \delta n_{x}$. The surface term

$$
F_{s}=2^{-1} A \int_{x_{0}}^{x_{0}+\lambda} e\left[\left(\delta n^{2}\right)_{z=0}+\left(\delta n^{2}\right)_{z=D}\right] d x
$$

assumes a boundary in which the minimum energy is with the director normal to the surfaces. The term $F_{s}^{\prime}$ reflects the fact that there could be a surface polarization that would couple directly to the external field. Since there is no physical way to separate that terms that arise from surface interactions from terms that are left over after Eq. (2.1) is converted into Eq. (2.2) by partial integration, we define a single phenomenological parameter $p$ that includes both. The term $F_{s}^{\prime}$ thus has the form

$$
F_{s}^{\prime}=-\int_{x_{0}}^{x_{0}+\lambda e} p\left[\left(\delta n E_{x}\right)_{z=0}-\left(\delta n E_{x}\right)_{z=D}\right] d x .
$$

The tacit assumption that the upper and lower surfaces are identical has been made. Minimizing the total free energy with respect to $\delta n$ at constant $\mathbf{E}$ obtains the volume equation

$$
K_{11} \frac{\partial^{2} n}{\partial x^{2}}+K_{33} \frac{\partial^{2} n}{\partial z^{2}}=f \frac{\partial E_{x}}{\partial z}-(4 \pi)^{-1} \Delta \epsilon^{0} E_{x} E_{z}
$$

and the boundary conditions

$$
\pm\left(K_{33} \frac{\partial n}{\partial z}+\rho \delta n E_{x}\right)+A \delta n=0,
$$

where the positive sign holds for the upper surface at $z=D$ and the negative sign for the lower surface. These equations are linear in on and the general solution can therefore be obtained as a linear superposition of solutions in which the flexoelectric and dielectric coupling are treated separately. Also solutions with different wave vector $q_{x}$ can be superposed to account for the nonsinusoidal nature of $\mathbf{E}$, or $\phi$. In any case one searches for solutions to equations of the following form:

$$
\begin{aligned}
& -\frac{\partial^{2} n}{\partial z^{2}}+q_{e}^{2} n=h \exp (-\kappa z), \\
& \frac{\partial n}{\partial z}+q_{s} n=h_{s}^{D} \quad \text { at } z=D, \\
& \frac{\partial n}{\partial z}-q_{s} n=h_{s}^{0} \quad \text { at } z=0
\end{aligned}
$$


where for flexoelectric coupling

$$
\begin{aligned}
& h=-K_{33}^{-1} f\left(\frac{\partial E_{x}\left(q_{x}\right)}{\partial z}\right)_{z=0^{+}}, \\
& q_{e}^{2}=K_{33}^{-1} K_{11} q_{x}^{2}, \\
& q_{s}=K_{33}^{-1} A \text {, } \\
& \kappa=\left(\epsilon_{\perp}^{0} / \epsilon_{11}^{0}\right)^{1 / 2} q_{x}, \\
& h_{s}^{D}=-K_{33}^{-1} p E_{x}\left(q_{x}\right)_{z=D},
\end{aligned}
$$

and

$$
h_{s}^{0}=-K_{33}^{-1} p E_{x}\left(q_{x}\right)_{z=0^{+}} .
$$

In practice the experiments have been done with voltages that were sinusoidal functions of time. The same equations hold except that $q_{e}^{2}$ is replaced by $q_{\omega}^{2}=q_{e}^{2}(1$ $\left.+j \omega \omega_{0}^{-1}\right)$, where $\omega_{0}=K_{11} q_{x}^{2}\left(\gamma_{1}^{*}\right)^{-1}$ and $\gamma_{1}^{*}$ is an effective viscosity parameter that describes the relaxation behavior of the nematic splay mode. Using the notation of Forster et al. ${ }^{6}$

$$
\left(\gamma_{1}^{*}\right)^{-1}=\gamma_{1}^{-1}+\frac{1}{4}(1-\lambda)^{2} \nu_{3}^{-1} \text {. }
$$

\section{Smectic phase}

The situation in the smectic phase is not significantly different from the nematic. In place of Eq. (4.6) the bulk contribution to the free energy is

$$
\begin{aligned}
F_{v}= & \frac{1}{2} \int_{x_{0}}^{x_{0}+\lambda_{e}} \int_{0}^{D} d z\left[B\left(\frac{\partial u}{\partial z}\right)^{2}+K_{11}\left(\frac{\partial^{2} u}{\partial x^{2}}\right)^{2}\right. \\
& \left.-2 f\left(\frac{\partial u}{\partial x}\right)\left(\frac{\partial E_{x}}{\partial z}\right)+(2 \pi)^{-1} \Delta \epsilon^{0} E_{x} E_{z} \frac{\partial u}{\partial x}\right] .
\end{aligned}
$$

Equation (4.12) is only applicable in the hydrodynamic approximation in which the director variation $\delta n_{x}$ is uniquely related to the layer displacement $-\nabla_{x} u$. Elsewhere in this paper we show data near the nematicsmectic phase transition where this may not be strictly true. However, we make this approximation here. In a similar vein we assume the boundary conditions can be given in terms of the layer displacement and take the surface energies as

$$
F_{s}=\frac{1}{2} A \int_{x_{0}}^{x_{0}+\lambda} e\left[\left(u^{2}\right)_{z=0}+\left(u^{2}\right)_{z=D}\right] d x
$$

and a surface polarization term

$$
F_{s}^{\prime}=-\int_{x_{0}}^{x_{0}+\lambda_{e}} p\left\{\left[\left(\frac{\partial u}{\partial x}\right) E_{x}\right]_{z=D}-\left[\left(\frac{\partial u}{\partial x}\right) E_{x}\right]_{x=0}\right\} d x
$$

The resultant differential equations are essentially identical to Eq. (4.11) with the changes

$$
\begin{aligned}
& h=-B^{-1} f q_{x}^{2}\left(\frac{\partial E_{x}\left(q_{x}\right)}{\partial z}\right)_{z=0^{+}}, \\
& q_{e}^{2}=B^{-1} K_{11} q_{x}^{4} \ll q_{x}^{2}, \\
& q_{s}=B^{-1} A, \\
& h_{s}^{D}=-B^{-1} p q_{x}^{2} E_{x}\left(q_{x}\right)_{z=D}, \\
& h_{s}^{0}=-B^{-1} p q_{x}^{2} E_{x}\left(q_{x}\right)_{z=0^{+} .}
\end{aligned}
$$

The solution to the time-dependent problem is trickier in the smectic case because at a finite ratio of $q_{a}$ $q_{x}$ the overdamped undulation or splaylike mode couples to the shear velocity so as to form an underdamped propagating shear mode. The conditions under which this actually happens depend on the relative magnitude of a number of different variables and we will not go into that here. Rather, we confine ourselves to the case that $q_{z}$ is small and assume a single overdamped nematiclike mode. In this case ${ }^{19}$ the time-dependent problem is approximately solved by the substitution $q_{\omega}^{2}=q_{e}^{2}(1$ $\left.+j \omega / \omega_{0}\right)$ where $\omega_{0} \approx K_{11} q_{x}^{2} / \nu_{3}$, where $\nu_{3}$ is a shear viscosity in the notation of Martin et al. ${ }^{\text {? }}$

\section{Solution for deformations}

The solution of Eq. (4.11) with boundary conditions is standard

$$
\begin{aligned}
\delta n(\omega)= & U \exp \left(-q_{\omega} z\right)+V \exp \left(+q_{\omega} z\right) \\
& +h\left(q_{\omega}^{2}-\kappa^{2}\right)^{-1} \exp (-\kappa z),
\end{aligned}
$$

where $q_{\omega}=q_{e}\left(1+j \omega / \omega_{0}\right)^{1 / 2}$. Setting

$$
\begin{aligned}
W= & \left(q_{s}+q_{\omega}\right)^{2} \exp \left(q_{\omega} D\right)-\left(q_{s}-q_{\omega}\right)^{2} \exp \left(-q_{\omega} D\right), \\
U= & W^{-1}\left\{\left[-h_{s}^{0}-h\left(\kappa+q_{s}\right)\left(q_{\omega}^{2}-\kappa^{2}\right)^{-1}\right]\left(q_{s}+q_{\omega}\right) \exp \left(q_{\omega} D\right)\right. \\
& -\left[h_{s}^{D}+h\left(\kappa-q_{s}\right)\left(q_{s}-q_{\omega}\right)\left(q_{\omega}^{2}-\kappa^{2}\right)^{-1} \exp (-\kappa D)\right\}, \\
V= & W^{-1}\left\{\left[h_{s}^{D}+h\left(\kappa-q_{s}\right)\left(q_{\omega}+q_{s}\right)\left(q_{\omega}^{2}-\kappa^{2}\right)^{-1} \exp (-\kappa D)\right]\right. \\
& \left.+\left(q_{s}-q_{\omega}\right)\left[h_{s}^{0}+h\left(\kappa+q_{s}\right)\left(q_{\omega}^{2}-\kappa^{2}\right)^{-1}\right] \exp \left(-q_{\omega} D\right)\right\} .
\end{aligned}
$$

If we maintain the tacit assumption that the electric fields, Eqs. (4.4), and thus the distortions are periodic and of infinite extent in $x$, the light scattering will only occur for specific values of $q_{x} \hat{x}=\mathrm{k}_{p}^{s}-\mathrm{k}_{p}^{i}=(4 n+1) \pi d^{-1} \hat{x}$. For each value of $q_{x}$ the amplitude of the scattered $E$ field will be shown below to depend on $q_{z}=k_{z}^{s}-k_{z}^{i}$ according to $\delta n\left(q_{z}\right)=\int_{0}^{D} \delta n(z) \exp \left(-i q_{z} z\right) d z$. Since the general expressions for $\delta n\left(q_{z}\right)$ are lengthy, we will not write them out, but rather concentrate on specific examples.

First, observe that $\kappa D \sim 5 \times 10^{2}$ and since $h_{s}^{D}$ $\sim \exp (-\kappa D)$, there is negligible error in taking $h_{s}^{D} \sim 0$. Second, in the nematic phase $q_{e} \sim q_{x} \sim 10^{4} \mathrm{~cm}^{-1}$ and since $A$ can be estimated ${ }^{20}$ to be of the order of $10^{-4} \mathrm{erg} \mathrm{cm}^{-2}$ or less, we obtain $q_{s} \sim 10^{2} \mathrm{~cm}^{-1}$. The result is that $\left|q_{\omega}\right| D \gg 1$ and also $\left|q_{\omega}\right| \gg q_{s}$. Furthermore, if we restrict experimental observations to small $q_{z}=k_{z}^{s}-k_{z}^{i}$ such that $K_{11} q_{x}^{2} \gg K_{33} q_{z}^{2}$,

$$
\delta n\left(q_{z}\right)=(f+p)\left(K_{11} q_{x}^{2}+j \gamma_{1}^{*} \omega\right)^{-1}\left[E_{x}\left(q_{x}\right)\right]_{z=0},
$$

where $\left[E_{x}\left(q_{x}\right)\right]_{z=0}$ is obtained from Eqs. (4.4) after substituting $z\left(\epsilon_{\perp}^{0} / \epsilon_{\| 1}^{0}\right)^{1 / 2}$ for $z$ by considering the coefficient of $\exp \left\{ \pm i\left[(4 n+1) \pi x d^{-1}\right]\right\}$ when $q_{x}=(4 n+1) \pi / d$. For the lowest-order diffraction effect, $n=0$ and

$$
\left[E_{x}\left(q_{x}\right)\right]_{z=0} \approx-2^{-1} \pi G^{-1} V d^{-1} .
$$

The prediction is that $\delta n\left(q_{z}\right)$ is relatively insensitive to $q_{z}$ in the nematic phase and its frequency dependence is essentially Lorentzian. It is also worth mentioning that, although the above numerical estimates result in a "free boundary condition" in which $A$ is neglected, these same values predict a "rigid boundary" in typical Frederickz geometries. Alternatively, a larger value for $A$ in which $q_{s} \gg q_{\omega}$ obtains

$$
\sin \left(q_{z}\right) \approx f\left[E_{x}\left(q_{x}\right)\right]_{z=0}\left[K_{33} q_{\omega}\left(q_{\omega}+\kappa\right)\right]^{-1} .
$$

As in the other example, Eq. $(4.18)$ predicts that $\delta n\left(q_{z}\right)$ 
is insensitive to $q_{z}=k_{z}^{s}-k_{z}^{i}$ so that the angular dependence of the scattering cannot be used to distinguish between the two cases. The predicted frequency dependence, however, is sufficiently different to distinguish between the two. Figure 10(a) described typical Lorentzian behavior in the nematic phase and we take this to confirm $q_{s} \ll q_{x}$ in the present geometry. Anticipating the results for the predicted relation between observed light scattering and $\delta n\left(q_{z}\right)$, the measurements described above should obtain a measure of $(f+p) K_{11}^{-1}$ in the nematic phase.

In the smectic phase the magnitudes are somewhat different. First, $q_{e}=\left(B^{-1} K\right)^{1 / 2} q_{x}^{2} \sim 10^{0}$ or $10^{1} \mathrm{~cm}^{-1}$. Second, the surface energy [Eq. (4.13)] involves constants $A$ that are either as large as the elastic constants of the cell walls or otherwise involve severe distortions of the smectic ordering near the walls. According to the definition, values of $q_{s}=B^{-1} A \geq q_{x} \approx 10^{4}-10^{5} \mathrm{~cm}^{-1}$ are not unreasonable. Thus, the smectic phase satisfies "rigid boundary conditions" in which $q_{s} \gg q_{e}$. Assuming that $q_{s}$ is also large compared with $q_{x}$

$$
\begin{aligned}
\delta n\left(q_{z}\right) \approx & -(\kappa)^{-2}\left(q_{\omega}^{2}+q_{z}^{2}\right)^{-1}\left[\sinh \left(q_{\omega} D\right)\right]^{-1} \\
& \times\left[q_{\omega} \exp \left(-i q_{z} D\right)-q_{\omega} \cosh \left(q_{\omega} D\right)+i q_{z} \sinh \left(q_{\omega} D\right)\right] \\
& \times B^{-1} f q_{x}^{2}\left(\frac{\partial E_{x}\left(q_{x}\right)}{\partial z}\right)_{z=0}
\end{aligned}
$$

In the limit that $\omega \rightarrow 0$ and $q_{z} \rightarrow 0$ this simplifies to

$$
\delta n\left(q_{z}\right) \approx(2 B)^{-1} f\left(\epsilon_{\| 1}^{0} / \epsilon_{\perp}^{0}\right)^{1 / 2} D q_{x}\left[E_{x}\left(q_{x}\right)\right]_{z=0} .
$$

The most striking difference between the results for the smectic and nematic phases is that $\operatorname{son}\left(q_{2}\right)$ is very sensitive to $q_{z}$ in the smectic phase, while it is relatively insensitive to the nematic. This arises from the extreme anisotropy of the smectic elasticity. In practice the $q_{z}$ dependence of Eq. (4.19) is sensitive to sample thickness and typical half-widths $\Delta q_{2}$ are of the order of $\pi / D$.

The frequency dependence predicted by Eq. (4.19) contains a "fall-off frequency" corresponding to $\left|q_{\omega}\right| D$ $\sim \pi$ or $\omega \sim \pi^{2} B\left(q_{x} D\right)^{-2} \nu_{3}^{-1}$. Typical numerical values obtain theoretical estimates of this fall-off frequency as $10^{4}$ $\mathrm{Hz}$.

\section{Light scattering amplitudes}

Although many authors have considered light scattering phenomena from both anisotropic media and dielectric slabs, we have not found explicit formulas for the examples discussed in this paper and we have had to derive the results ourselves. The methods are straightforward and in lieu of a step-by-step derivation we will just outline the procedure.

Using the results of Secs. II-IV B we assume an optical inhomogeneity $\delta \epsilon_{x z}$ linearly related to $\delta$. For a given incident optical electric field, there is an inhomogeneous optical polarization $\delta \mathbf{P}=(4 \pi)^{-1} \delta \epsilon \cdot \mathbf{E}$. We now search for solutions to

$$
\begin{aligned}
& \nabla \times \mathbf{E}=-c^{-1} \frac{\partial \mathbf{B}}{\partial t}, \\
& \nabla \times \mathbf{B}=c^{-1} \boldsymbol{\varepsilon} \cdot \frac{\partial \mathbf{E}}{\partial t}+c^{-1} 4 \pi \frac{\partial \delta \mathbf{P}}{\partial t},
\end{aligned}
$$

$$
\begin{aligned}
& \boldsymbol{\nabla} \cdot(\boldsymbol{\epsilon} \cdot \mathbf{E}+4 \pi \delta \mathbf{P})=\mathbf{0}, \\
& \boldsymbol{\nabla} \cdot \mathbf{B}=0,
\end{aligned}
$$

consistent with the appropriate boundary conditions on the anisotropic dielectric slab. The solutions are approximate only in the sense that they do not include the effects of either reflected or multiple scattered beams in the fields that give rise to the $\delta P$ term. Multiple scattering effects would not be linear in $\delta \epsilon$ and their neglect is not serious. Adequate formulas to include effects due to multiple reflections of the incident laser beam would result in small corrections. Similarly multiple reflection corrections for the scattered radiation are also neglected. The present problem is simplified by the conservation of the wave vector in the $x y$ plane. The general solution for the $E$ and $M$ wave outside the dielectric slab has both a fixed $k_{p}^{s}=k_{p}^{i}+q_{x}$ and $\left|k^{s}\right|$. These in turn fix $k_{z}^{s}$. Thus we assume that for each polarization there is one plane wave for arbitrary amplitude and phase propagating away from the dielectric slab above the slab and a second one below. Inside the slab we assume the $E$ and $M$ wave is the superposition of one plane wave with fixed $k_{p}^{s}$ and $k_{z}^{s}>0$ and a second with $k_{z}^{s}<0$ that both satisfy the homogeneous Maxwell's equations plus an "inhomogeneous" solution. The inhomogeneous solution is facilitated by expressing $\delta P(z)$ as a Fourier series in $\exp [ \pm i(n \pi z / D)]$. The only subtle point is to ensure that the requirement $\nabla \cdot \mathbf{E}$ $+4 \pi \delta \mathrm{P})=0$ is satisfied for the inhomogeneous solution. This, however, is a standard problem in nonlinear optics. ${ }^{21}$ Together with application of the usual $E$ and $M$ boundary conditions, this procedure reduces the problem to a linear $4 \times 4$ matrix problem in which the complex amplitudes of the four optical electric fields are determined. Recalling the tacit assumption that $\delta n(x)$ is both periodic and of infinite extent along $x$, the quantity $\operatorname{\delta n}\left(q_{z}\right)$ appearing in the following results for the amplitude of the scattered $E$ fields is defined such that for specific $\mathrm{k}_{p}^{s}-\mathrm{k}_{p}^{i}=q_{x} \hat{x}$, $\delta n\left(q_{z}\right)$ is the coefficient of $\exp \left(+i q_{x} x\right)$ in the expression for $\int_{0}^{D} n(x, z) \exp \left(-i q_{z} z\right) d z$. This is consistent with a sign convention that takes the $\mathbf{E}$ fields to be the real part of waves $\exp [-i(\omega t-\mathrm{k} \cdot \mathrm{r})]$.

\section{Incident beam-ordinary polarization}

The scattered wave is entirely extraordinary with amplitude

$$
E^{s}=\frac{i k^{0} k_{p}^{s}\left(\epsilon_{\perp} / \epsilon_{11}\right) \Delta \epsilon \delta n\left(q_{g}\right) \sin \alpha^{l}}{k_{z}^{s}+\epsilon_{\perp} k_{z}^{\text {ext }}} E^{i},
$$

where $\mathbf{k}^{\text {ext }}$ is the wave vector for the scattered light outside the dielectric, $k^{0}=2 \pi \lambda_{0}^{-1}$, and $E^{i}$ is the incident optical electric field. Geometric considerations obtain

$$
\begin{aligned}
q_{z}= & k_{z}^{s}-k_{z}^{i} \\
= & \left\{k_{\perp}^{2}-\left(\epsilon_{\perp} / \epsilon_{11}\right)\left[\left(k_{p}^{i}\right)^{2}+q_{x}^{2}+2 k_{p}^{i} q_{x} \cos \alpha^{i}\right]\right\}^{1 / 2} \\
& -\left[k_{\perp}^{2}-\left(k_{p}^{i}\right)^{2}\right]^{1 / 2},
\end{aligned}
$$

where $k_{\perp}^{2}=\epsilon_{\perp} k_{0}^{2}$ and $k_{\|}^{2}=\epsilon_{\sharp} k_{0}^{2}$. The results previously obtained indicate that large-amplitude will be observed in the smectic phase only near $q_{z}=0$. This obtains

$$
\cos \alpha^{i}=2^{-1}\left[k_{p}^{i} q_{x}^{-1}\left(\epsilon_{11} \epsilon_{\perp}^{-1}-1\right)-q_{x}\left(k_{p}^{i}\right)^{-1}\right]
$$

The most convenient experimental geometry was $\alpha^{i}$ 
$\approx \frac{1}{2} \pi$ or $q_{x} \approx k_{p}^{i}\left(\epsilon_{\| 1} \epsilon_{1}^{-1}-1\right)^{1 / 2}$. This maximized the scattered intensity and kept the algebra as simple as possible. Variation of $\alpha^{i}$ at constant $\beta^{i}$ also varies $q_{z}$ and for small angles;

$$
\delta q_{z}=\left(\epsilon_{\perp} / \epsilon_{11}\right) q_{x}\left[k_{\perp}^{2}\left(k_{p}^{i}\right)^{-2}-1\right]^{-1 / 2} \sin \alpha^{i} \delta \alpha^{i} .
$$

Typical numerical values obtain $\delta q_{z} / q_{z} \approx 10^{-2}$ for $\delta \alpha$ $\approx 0.05 \mathrm{rad}$ and demonstrate that the values of $q_{z}$ can be set with relatively high precision.

\section{E. Incident beam with extraordinary polarization}

Scattered light is observed in both ordinary and extraordinary polarizations. The result for the ordinary polarization (i.e., the depolarized light scattering) is

$$
\begin{aligned}
E^{s}= & i k_{0}^{2} k_{p}^{i}\left(k_{z}^{\mathbf{x x t}}+k_{z}^{s}\right)^{-1}(\Delta \epsilon) \epsilon_{\perp} \sin \beta^{i} \sin \alpha^{i} \\
& \times \delta n\left(q_{z}\right) E^{i}\left(\epsilon_{\perp}^{2} \sin ^{2} \beta^{i}+\epsilon_{\|}^{2} \cos ^{2} \beta^{i}\right)^{-1 / 2} \\
& \times\left[\left(k_{p}^{i}\right)^{2}+q_{x}^{2}+2 q_{x} k_{p}^{i} \cos \alpha^{i}\right]^{-1 / 2}
\end{aligned}
$$

with

$$
\begin{aligned}
q_{z}= & k_{z}^{s}-k_{z}^{i} \\
= & {\left[k_{\perp}^{2}-\left(k_{p}^{i}\right)^{2}-q_{x}^{2}-2 k_{p}^{i} q_{x} \cos \alpha^{i}\right]^{1 / 2} } \\
& -\left[k_{\perp}^{2}-\left(\epsilon_{\perp} / \epsilon_{\| 1}\right)\left(k_{p}^{i}\right)^{2}\right]^{1 / 2} .
\end{aligned}
$$

In our samples $\epsilon_{\|}>\epsilon_{1}$ and the condition for $q_{z} \approx 0$

$$
\cos \alpha^{i} \approx k_{p}^{i}\left(\epsilon_{\perp} \epsilon_{\| 1}^{-1}-1\right)\left(2 q_{x}\right)^{-1}-q_{x}\left(2 k_{p}^{i}\right)^{-1}
$$

is only satisfied if $\cos \alpha^{i}<0$ or $\alpha^{i}>\frac{1}{2} \pi$. The maximum intensity obtained from Eq. (4.26) is smaller than obtainable from Eq. (4.22) because in the present case $\alpha$ $=\frac{1}{2} \pi$ cannot be obtained. Although we did not make extensive measurements to verify the equations, typical intensities were found smaller for this geometry as compared to the intensity in the geometry appropriate to Eq. (4.22). Note also that both Eqs. (4.22) and (4.26) are proportional to $\sin \alpha^{i}$. The qualitative effects of this factor are easily observed in either geometry.

With both incident and scattered light of extraordinary polarization, the theoretical result for the scattered amplitude is

$$
\begin{aligned}
E^{s}= & i k_{0} \epsilon_{\perp}\left(k_{z}^{s}+\epsilon_{\perp} k_{z}^{\text {ext }}\right)^{-1} \Delta \epsilon \delta n\left(q_{z}\right) E^{i} \\
& \times\left(k_{z}^{s} \sin \beta^{i} \cos \gamma+k_{p}^{s} \cos \beta^{i} \cos \alpha^{i}\right) \\
& \times\left(\epsilon_{\perp}^{2} \sin ^{2} \beta^{i}+\epsilon_{\|}^{2} \cos ^{2} \beta^{i}\right)^{-1 / 2}
\end{aligned}
$$

where

$$
\cos \gamma=\left(q_{x}+\left|\mathbf{k}_{p}^{i}\right| \cos \alpha^{i}\right)\left(\left|\mathbf{k}_{p}^{i}\right|^{2}+q_{x}^{2}+2\left|\mathbf{k}_{p}^{i}\right| q_{x} \cos \alpha^{i}\right)^{-1 / 2} .
$$

With a small amount of algebra, Eq. (4.28) can be shown to have the form $E^{s} \sim\left(q_{z} / q_{x}\right) \delta n$. The intensity ratio of polarized to depolarized scattering when the incident light is extraordinarily polarized is roughly $\left(q_{z} / q_{x}\right)^{2}$. In the smectic phase $\delta n\left(q_{z}\right)$ is sharply peaked at $q_{z} \sim 0$ and the two effects combine such that polarized scattering is considerably weaker than depolarized. Typically, scattering can be observed at small nonzero values of $q_{z} \sim \pi / D$, and for $D \sim 500 \mu \mathrm{m}$ and $2 \pi q_{x}^{-1} \approx 5 \mu \mathrm{m}$ the ratio of polarized to depolarized intensities is of the order of $10^{-4}$. Polarized scattering is clearly not important in the smectic phase.

In the nematic phase this geometry does have practi- cal application. For one thing $q_{z}$ and $q_{x}$ can be of comparable magnitude so the intensity ratio is not as unfavorable for polarized scattering as in the smectic. Second, as we mentioned earlier, the experimental electrodes do not provide voltages $\phi(x, z)$ that are truly sinusoidal in the $x$ direction. Furthermore, the electrodes themselves act as a diffraction grating and scatter the incident light into a number of different Bragg orders. At any single Bragg angle one thus observes the desired scattering of the incident light by one preselected Fourier component of $\delta \epsilon_{x z}$ plus a background that consists of light first scattered by the metallic grating and second scattered by a different Fourier component of $\delta \epsilon_{x z}$. These multiplie scattering effects can be separated from the principal one in which the incident beam $\mathrm{k}^{i}$ is scattered into the detector by the fundamental Fourier component of $\delta \epsilon\left(q_{x}\right)$ if one chooses the angles $\alpha^{i}, \beta^{i}, \alpha^{s}$, and $\beta^{s}$ such that $q_{z}$ is near zero for this Fourier component. The resultant scattered intensity then has the form $\left|m q_{z}+m^{\prime}\right|^{2}$, where $m q_{z}$ is obtained from Eq. (4.28) for the principal diffraction and $m^{\prime}$ is the background due to other orders. Data demonstrating this effect is shown in Fig. 7 for the nematic phase and in Fig. 8 for the smectic. Note that in the smectic $m^{\prime} \approx 0$ as expected. In order to evaluate the experimental ratio $f / K_{11}$ in the nematic phase, we used this effect.

\section{QUANTITATIVE RESULTS}

\section{A. Nematic phase}

One result of the theoretical analysis in Sec. IV was the recognition that the $q_{z}$ dependence of the flexoelectric signal that was displayed in Fig. 7 can be used to separate higher-order diffraction effects from the principle first-order effect. The relevant theoretical formula is Eq. (4.28). In the special case that $\alpha^{i}=\pi$ and $\alpha^{s}$ $=0$, with the angles $\beta^{i}$ and $\beta^{s}$ both small [of magnitude $q_{x}\left(2 k_{\perp}\right)^{-1}$ or smaller], and both waves polarized as extraordinary waves, several approximations are possible.

The most subtle involves $k_{z}^{s} \sin \beta^{i} \cos \gamma+k_{x}^{s} \cos \beta^{i} \cos \alpha^{i}$ which is rigorously equal to $\left|\mathbf{k}^{s}\right| \sin \left(\beta^{i}-\beta^{s}\right)$ when $\cos \gamma$ $=1$ and $\cos \alpha^{i}=-1$. For small angles and near to the condition $q_{z}=0, q_{z}=k_{z}^{s}-k_{z}^{i} \approx 2^{-1}\left(\epsilon_{1} / \epsilon_{i n}\right)\left|k_{1}\right|\left[\left(\beta^{i}\right)^{2}-\left(\beta^{s}\right)^{2}\right]$ and since $q_{x} \approx\left|\mathbf{k}^{s}\right|\left(\beta^{s}+\beta^{i}\right)$, these can be combined to ob$\operatorname{tain} \beta^{i}-\beta^{s} \approx 2\left(\epsilon_{\mathfrak{i}} / \epsilon_{1}\right)\left(q_{z} / q_{x}\right)$. With the other obvious approximations

$$
\left(E^{i}\right)^{-1} E^{s} \approx-i 2 k_{0}\left[1+\left(\epsilon_{1}\right)^{1 / 2}\right]^{-1} \Delta \epsilon \delta n\left(q_{z}\right) q_{z} q_{x}^{-1} .
$$

For experimental purposes it is more convenient to express this result in terms of the value for $\beta^{i}$ outside the material $\left(\beta^{i}\right)^{\text {ext }} \approx\left(\epsilon_{\perp}\right)^{1 / 2} \beta^{i}$ and since $\delta \beta^{i}$ must equal $-\delta \beta^{s}$ if $q_{x}$ is fixed, we can write $\left(\delta \beta^{i}\right)^{\text {ext }} \approx+\epsilon_{11} \epsilon_{\perp}^{-1 / 2}\left(q_{z} / q_{x}\right)$ and

$$
\left(E^{i}\right)^{-1} E^{s} \approx-i 2 k_{0} \epsilon_{1}^{1 / 2} \epsilon_{\| 1}^{-1}\left[1+\left(\epsilon_{1}\right)^{1 / 2}\right]^{-1} \Delta \epsilon \delta n\left(q_{z}\right)\left(\delta \beta^{i}\right)^{\text {ext }} \text {. }
$$

Using Eq. (4.17a) with $\omega \ll K_{11} q_{x}^{2} / \gamma_{1}^{*}$ and Eq. (4.17b) with $q_{z} \ll \kappa$,

$$
\begin{aligned}
\left(E^{i}\right)^{-1} E_{s}= & i k_{0} \epsilon_{\perp}^{1 / 2} \epsilon_{\| 1}^{-1}\left[1+\left(\epsilon_{\perp}\right)^{1 / 2}\right]^{-1} \Delta \epsilon(f+p) \\
& \times\left(K_{11} q_{x}\right)^{-1} V G^{-1}\left(\delta \beta^{i}\right)^{\text {ext }}
\end{aligned}
$$

It is straightforward to demonstrate that if $I_{1}(2 \omega)$ is the 
integrated intensity at the appropriate Bragg spot, this expression for $\left(E^{i}\right)^{-1} E^{s}$ is equal to $\left[2 I_{1}(2 \omega) / I_{0}\right]^{1 / 2}$ where the factor of 2 arises from the fact that $I_{1}(2 \omega)$ is the temporal average of the square of a sinusoidal function of time. The experiment was performed at $T-T_{\mathrm{NA}}$ $\approx 1{ }^{\circ} \mathrm{C}$ where $T_{\mathrm{NA}} \approx 34.65^{\circ} \mathrm{C}$ and the observed slope was $\left[\left(\delta \beta^{l}\right)^{\text {ext }}\right]^{-1}\left[2 I_{1}(2 \omega) / I_{0}\right]^{1 / 2}=0.2$ for $V=1 \mathrm{~V}$. The other parameters in Eq. (5.3) can be obtained from Refs. 15 and 16. In particular with $K_{11}=1.9 \times 10^{-6}$ dyn, the deduced value of $f+p=2.5 \times 10^{-4}$ esu cm$^{-1}$. Further, if we assume $f \gg p$ as previously argued on the basis of the screening effects observed below a few $\mathrm{Hz}$, this is the experimental value of $f$. Both previous experimental results for $\mathrm{MBBA}^{3}$ and theoretical estimates of $e_{11}$ and $e_{33}{ }^{1,10}$ obtained similar magnitudes. The uncertainty in the present numerical result could be as large as 40$50 \%$ if one includes all of the independent possibilities for error. Note also that the maxima tilt angle $\delta n(x, z)$ in these experiments was only of the order of $2^{\circ}$ and this should be well within the range in which the linear theory is applicable. For CBOOA the magnitude of $f$ was also of the order of $10^{-4}$ esu $\mathrm{cm}^{-1}$.

We can also give a tentative assignment to the sign of $f+p$ for BBMBA. For the geometry appropriate to Fig. 7 , the electromagnetic field scattered by the flexoelectric effect can be assigned a phase from Eq. (5.3). Note that the phase appearing in Eq. (5.3) is dependent on (1) the convention in which $E$ is given in terms of the real part of complex waves that propagate as $\exp [-i(\omega t-\mathbf{k} \cdot \mathbf{r})$ and (2) the origin of the $x$ axis relative to the electrode position. For example, Fig. 16 specifies the origin of the $x$ axis as coinciding with the center of the space between the two electrodes. For a positive voltage $V$, the amplitude and phase of the fundamental components in the Fourier series given by Eqs. (4.4) are fixed. A shift of origin from between the electrodes to the center of an electrode would convert the cosine series for $E_{x}$ into a sine series and the coefficient of $\exp \left[i\left(\pi x d^{-1}\right)\right]$ in that series would differ from the coefficient in the cosine series by a factor of $i$. The phase factors in Eq. (5.1) result from these conventions as well as the physical effect of flexoelectric phase modulation of the incident light.

As discussed above, heterodyne detection of the lowest-order flexoelectric signal is possible because near the ends the metallic electrode pattern has the same periodicity as the flexoelectric deformation. Although the flexoelectric deformation produces a phase modulation, the scattering due to the opaque electrodes is the result of spatially amplitude modulating the incident light. If the symmetries of the spatial amplitude and phase modulation relative to origin of the $x$ axis were identical, the two signals would be $\frac{1}{2} \pi$ out of phase and heterodyning would not occur. In fact the symmetries are not the same. The phase modulation is symmetric about the space between electrodes, while the amplitude modulation is symmetric about the electrode center. Flexoelectric phase modulation is described by a cosine series in $x$ with imaginary amplitudes, while the spatial amplitude modulation from the ends of the electrodes is described by a sine series in $x$ with real amplitudes. For a particular scattering direction the coefficients of $\exp \left(i q_{x} x\right)$ are both purely imaginary.
Of particular interest is the different symmetries from the ends of the electrode at $y>0$ and $y<0$. The transmission functions from the two ends are identical except that one is shifted by $d$ relative to the other. For $q_{x}=\pi / d$ this means that the phase of the heterodyne signal relative to the voltage $V$ will differ by $\pi$ depending on whether the reference signal is taken from the end of the electrodes with $y>0$ or $y<0$. This effect has been observed. The optical phase angle $\phi$ predicted by this calculation is either 0 or $\pi$. In practice, the observed angle in the nematic phase typically satisfied $|\cos \phi|$ $\approx 0.7$. Further, by comparing the relative phase of the heterodyne signal and applied voltage with the phase predicted by the above analysis, we conclude that $f+p$ $>0$. Since we previously argued $f \gg p$ on the basis of the low-frequency effects, this implies $f>0$.

\section{B. Smectic phase}

For $T \ll T_{\mathrm{NA}}$ the smectic flexoelectric signal was too small to be studied quantitatively by homodyne detection and we had to rely exclusively on the heterodyne method. The experimental geometry used $\alpha^{i}=90^{\circ}$ and $\alpha^{s}=0^{\circ}$ and we take

$$
I_{1}(\omega)=2^{-1}\left|E^{s}\right|\left|E^{r e f}\right| \cos \phi .
$$

The optical phase angle $\phi$ was chosen to satisfy $|\cos \phi|$ $=0.7$. This value was consistently found over a range of temperatures and angles $\beta^{i}, \alpha^{i}$ whenever both heterodyne and homodyne signals could be measured simultaneously. At room temperature, with $\beta^{i}=0.24, \alpha^{i}$ $=\frac{1}{2} \pi \mathrm{rad}$, and $V=2 \mathrm{~V}$, we obtained a value of $\left|E^{s}\right|\left|E^{i}\right|^{-1}$ $\sim 3.5 \times 10^{-6}$. Using $\mathrm{Eq}$. (4.22), the following numerical result is obtained: $\delta n\left(q_{z}\right) \approx 2.6 \times 10^{-11} \mathrm{~cm}$ for $q_{z} \approx 0$. This number can be inserted into Eq. (4.20) and with $\left[E_{x}\left(q_{x}\right)\right]_{z=0}=-2^{-1} \pi K^{-1} V d^{-1}$ [note that $E_{x}\left(q_{x}\right)$ is defined to be the coefficient of $\exp \left( \pm i \pi x d^{-1}\right)$ in the series defined in Eqs. (4.4)], one obtains $f / B \approx 3 \times 10^{-15} \mathrm{esu} \mathrm{cm}^{2} \mathrm{erg}^{-1}$. If we take $B \sim 10^{10} \mathrm{erg} \mathrm{cm}^{-3}$, we obtain $f \sim 3.5 \times 10^{-4}$ esu cm $\mathrm{cm}^{-1}$ in agreement with the value obtained for the nematic phase. Although this number is less precise than the nematic value because of the extra uncertainty associated with the optical heterodyne efficiency (i.e., the value of $\cos \phi$ ) and the fact that the range of possible values for $B$ is greater than for $K$, it is quite clear that its magnitude is not significantly different from the nematic value. Assuming the director tilt is simply related to the layer displacement, the above value of $\delta n\left(q_{z}\right)$ corresponds to a layer displacement of the order of $\sin \left(q_{z}\right)\left(D q_{x}\right)^{-1} \sim 2 \times 10^{-6} \AA$.

\section{SUMMARY}

The most important result of this paper is the unambiguous proof of the existence of flexoelectricity as a bulk phenomena in both nematic and smectic liquid crystals. Although earlier experiments were consistent with bulk flexoelectricity, the nature of these experiments was such that alternative interpretations could not be discounted. In particular, Helfrich's experiment ${ }^{3}$ could have been interpreted as a pure surface effect.

A second result is the demonstration that the flexoelectric coefficient $f=e_{11}+e_{33}$ does not depend on temperature in the manner that has previously been 
suggested on theoretical grounds. In fact, $f$ is essentially the same in the nematic and smectic phases of BBMBA. This is particularly striking in that it implies significant flexoelectric effects in regions of large smectic curvature like focal conic lines and small smectic vesicles. De Gennes makes the point in his work on liquid crystals that, to the extent flexoelectricity is a strong effect, the conventional local elastic theories are not correct. Of course for most macroscopic elastic experiments, flexoelectric effects are probably weak enough that they can be forgotten and both the Frank theory of nematics and the conventional elastic theories of smectics are valid. There are, however, regions of large curvature near the core of disclinations and smectic focal conic lines where flexoelectric effects may not be ignored. For example, consider a disclination where the director $\hat{n}=(x \hat{x}+y \hat{y})\left(x^{2}+y^{2}\right)^{-1 / 2}$. Assuming there is no free charge, one can demonstrate the existence of an electric field $E \sim f\left(x^{2}+y^{2}\right)^{-1 / 2}$. Taking $f \sim 10^{-4} \mathrm{esu} / \mathrm{cm}$ and a radius $\left(x^{2}+y^{2}\right)^{+1 / 2} \sim 10^{-6} \mathrm{~cm}$ obtains an electric field of $10 \mathrm{esu} / \mathrm{cm}^{2}=3 \times 10^{4} \mathrm{~V} / \mathrm{cm}$. It is not clear that no free charge and thus $\nabla \cdot D=0$ is the most realistic condition and if one allows charges to build up, the material near the disclination obviously will have a different impurity content than els ewhere in the sample. Since compositional inhomogeneity can have significant elastic effects, the effect of flexoelectricity could significantly modify calculations that attempt an estimation of the "core" energy of defects.

Helfrich has also pointed out the role that curvature elasticity can have on the shape of red blood cell erthrocytes. ${ }^{22,23}$ In view of the fact that the flexoelectric coefficient in smectic BBMBA is of the same magnitude as in nematic BBMBA, we are led to ask if the magnitude of the flexoelectric coefficient in lyotropic smectics (i.e., lamina phases of lipids) might not lead us to a flexoelectric origin for red blood cell curvature. Experiments exist that demonstrate that the outer and inner surfaces of erthrocytes have different biochemical properties. ${ }^{24}$ Other experiments have also demonstrated that charged ions like $\mathrm{Ca}^{++}$have specific effects on the shape of erthrocytes and thus the curvature of red blood cells membranes. ${ }^{25-28}$ Although there is no clear evidence that flexoelectric phenomena play a significant role in these effects, the symmetry in these problems is such that it could. We believe experiments on flexoelectricity in lamina lipid phases should be undertaken in order to evaluate the flexoelectric magnitudes.

\section{ACKNOWLEDGMENTS}

One of the authors (JP) acknowledges the friendly atmosphere he has found during his stay at Harvard University. It is also a pleasure to mention the gracious- ness with which S. Bernacki and H. Smith of MIT's Lincoln Laboratory have provided us with interdigital electrodes.

*Work supported in part by the Joint Services Electronics Program (U.S. Army, U.S. Navy, and U.S. Air Force) under Contract No. N00014-75-C-0648 and by the National Science Foundation under Grant No. DMR 72-03020-A05 and DMR 72-02 088.

'Permanent address: Centre de Recherche Paul Pascal, CNRS, Domaine Universitaire, 33405, Talence, F rance.

${ }^{1}$ R. B. Meyer, Phys. Rev. Lett. 22, 918 (1969).

${ }^{2}$ W. Haas, J. Adams, and J. B. Flannery, Phys, Rev. Lett. $25,1326(1970)$.

${ }^{3}$ D. Schmidt, M. Schadt, and W. Helfrich, Z, Naturforsch. A 27, 277 (1972).

${ }^{4}$ P. G. De Gennes, The Physics of Liquid Crystals (Clarendon, Oxford, 1974).

${ }^{5}$ P. C. Martin, P.S. Pershan, and J. Swift, Phys. Rev. Lett. $25,844(1970)$.

${ }^{6}$ D. Forster, T.C. Lubensky, P.C. Martin, J. Swift, and P.S. Pershan, Phys. Rev. Lett. 26, 1016 (1971).

${ }^{7}$ P.C. Martin, O. Parodi, and P.S. Pershan, Phys. Rev。A A 6, 2401 (1972).

${ }^{\circ}$ R. B. Meyer, L, Liebert, L. Strelecki, and P. Keller, J. Phys. Lett. 36, L69 (1975).

${ }^{9}$ P. G. De Gennes, J. Phys. Suppl. 30, C4 (1969).

${ }^{10} \mathrm{~W}$. Helf rich, Z. Naturforsch. A 26, 833 (1971).

${ }^{11}$ N. A. Clark and P.S. Pershan, Phys. Rev. Lett. 30, 3 (1973).

${ }^{12}$ G. Durand, C.R. Acad. Sci. (Paris) 275, 629 (1972).

${ }^{13} \mathrm{~J}$. Prost and H. Gasparoux, Phys. Lett. A 36, 245 (1971).

${ }^{14} \mathrm{~F}$. Rondelez, Solid State Commun. 14, 815 (1974).

${ }^{15} \mathrm{~L}$. Cheung and R.B. Meyer, Phys. Lett. A 43, 261 (1973).

See also L. Cheung, Ph. D. thesis (Harvard University, 1973) (unpublished).

${ }^{16}$ D. Dolphin, Z. Muljiani, J. Cheng, and R. B. Meyer, J. Chem. Phys. 58, 413 (1973).

${ }^{17}$ P.S. Pershan and J. Prost, J. Appl. Phys. 46, 2343 (1975).

${ }^{18}$ George $F$. Carrier, Max Krook, and Carl E. Pearson,

Functions of a Complex Variable: Theory and Technique (MeGraw-Hill, New York, 1966).

${ }^{19}$ P. G. De Gennes, Phys. F luids 17, 1645 (1974).

${ }^{20}$ W. Helfrich, Phys. Lett. A 35, 393 (1971).

${ }^{21} \mathrm{~N}$. Bloembergen, Nonlinear Optics (Benjamin, New York, 1965).

${ }^{22}$ W. Helfrich, Z. Naturforsch, C 28, 693 (1973).

${ }^{23} \mathrm{~W}$. Helf rich, H. J. Deuling, J. Phys. (Paris) (to be published).

${ }^{24} \mathrm{~A}$ general review of this problem is contained in Carl Martin Cohen, Ph. D. thesis (Harvard University, 1974), (unpublished).

${ }^{25}$ R. I. Weed, R, L. LaCelle, and E.W. Merrill, J. Chin. Invest. 48, 795 (1969).

${ }^{26}$ P. L. La Celle, F. H. Kirkpatrick, M. P. Udkow, and B. Arkin in Red Cell Shape, edited by M. Bessis, R. I. Weed, and P.F. LeBlond (Springer-Verlag, New York, 1973).

${ }^{27}$ P. L. LaCelle, F.H. Kirkpatrick, and M. P. Udkow in Erthrocytes, Thrombocytes and Leukocytes, edited by $\mathrm{E}$. Gerlack, K. Moser, K. Deutch, and W. Williams (Georg Theime, Stuttgart, 1973).

${ }^{28}$ R. I. Weed and B. Chailey in Red Cell Shape, edited by M. Bessis, R.I. Weed, and P.P.F. LeBlond (Springer-Verlag, New York, 1973), p. 55. 